\title{
Ankyrin-B dysfunction predisposes to arrhythmogenic cardiomyopathy and is amenable to therapy
}

Jason D. Roberts, ${ }^{1,2,}$ Nathaniel P. Murphy, ${ }^{3,4}$ Robert M. Hamilton, ${ }^{5}$ Ellen R. Lubbers, ${ }^{3,4}$ Cynthia A. James, ${ }^{6}$ Crystal F. Kline, ${ }^{3,4}$ Michael H. Gollob, ${ }^{7}$ Andrew D. Krahn, ${ }^{8}$ Amy C. Sturm, ${ }^{9}$ Hassan Musa, ${ }^{3}$ Mona El-Refaey, ${ }^{3}$ Sara Koenig, ${ }^{3}$ Meriam Åström Aneq, ${ }^{10}$ Edgar T. Hoorntje, ${ }^{11,12}$ Sharon L. Graw, ${ }^{13}$ Robert W. Davies, ${ }^{14}$ Muhammad Arshad Rafiq, ${ }^{5,15}$ Tamara T. Koopmann, ${ }^{5}$ Shabana Aafaqi, ${ }^{5}$ Meena Fatah, ${ }^{5}$ David A. Chiasson, ${ }^{16}$ Matthew R.C. Taylor, ${ }^{13}$ Samantha L. Simmons, ${ }^{3,4}$ Mei Han, ${ }^{3,4}$ Chantal J.M. van Opbergen, ${ }^{17}$ Loren E. Wold, ${ }^{3,4}$ Cianfranco Sinagra, ${ }^{18}$ Kirti Mittal, ${ }^{5}$ Crystal Tichnell, ${ }^{6}$ Brittney Murray, ${ }^{6}$ Alberto Codima, ${ }^{19}$ Babak Nazer, ${ }^{20}$ Duy T. Nguyen, ${ }^{21}$ Frank I. Marcus, ${ }^{22}$ Nara Sobriera, ${ }^{23}$ Elisabeth M. Lodder, ${ }^{24}$ Maarten P. van den Berg, ${ }^{25}$ Danna A. Spears, ${ }^{7}$ John F. Robinson, ${ }^{26}$ Philip C. Ursell, ${ }^{27}$ Anna K. Green, ${ }^{28}$ Allan C. Skanes, ${ }^{1}$ Anthony S. Tang, ${ }^{1}$ Martin J. Gardner, ${ }^{29}$ Robert A. Hegele, ${ }^{26,30}$ Toon A.B. van Veen, ${ }^{17}$ Arthur A.M. Wilde, ${ }^{24}$ Jeff S. Healey, ${ }^{31}$ Paul M.L. Janssen, ${ }^{3,4}$ Luisa Mestroni, ${ }^{13}$ J. Peter van Tintelen, ${ }^{12,32,33}$ Hugh Calkins, ${ }^{6}$ Daniel P. Judge, ${ }^{6,34}$ Thomas J. Hund, ${ }^{3,35}$ Melvin M. Scheinman, ${ }^{2}$ and Peter J. Mohler ${ }^{3,4}$

'Section of Cardiac Electrophysiology, Division of Cardiology, Department of Medicine, Western University, London, Ontario, Canada. ${ }^{2}$ Section of Cardiac Electrophysiology, Division of Cardiology, Department of Medicine, UCSF, San Francisco, California, USA. ${ }^{3}$ Dorothy M. Davis Heart and Lung Research Institute, The Ohio State University Wexner Medical Center, Columbus, Ohio, USA. ${ }^{4}$ Departments of Physiology and Cell Biology and Internal Medicine, Division of Cardiovascular Medicine, The Ohio State University Wexner Medical Center, Columbus, Ohio, USA. ${ }^{5}$ The Labatt Family Heart Centre (Department of Pediatrics) and Translational Medicine, The Hospital for Sick Children and the University of Toronto, Toronto, Ontario, Canada. ${ }^{6}$ Division of Cardiology, Department of Medicine, Johns Hopkins University, Baltimore, Maryland, USA. 'Peter Munk Cardiac Centre, Toronto General Hospital, University of Toronto, Toronto, Ontario, Canada. ${ }^{8}$ Heart Rhythm Services, Division of Cardiology, Department of Medicine, University of British Columbia, Vancouver, British Columbia, Canada. ${ }^{9}$ Cenomic Medicine Institute, Geisinger, Danville, Pennsylvania, USA. ${ }^{10}$ Department of Clinical Physiology and Department of Medical and Health Sciences, Linköping University, Linköping, Sweden. "Netherlands Heart Institute, Utrecht, Netherlands. ${ }^{2}$ Department of Cenetics, University Medical Center Groningen, University of Groningen, Groningen, Netherlands. ${ }^{3}$ Cardiovascular Institute and Adult Medical Genetics Program, University of Colorado Denver, Aurora, Colorado, USA. ${ }^{14}$ Program in Genetics and Genome Biology and The Centre for Applied Cenomics, The Hospital for Sick Children, Toronto, Ontario, Canada. ${ }^{15} \mathrm{Department} \mathrm{of} \mathrm{Bioscience,} \mathrm{COMSATS} \mathrm{University,} \mathrm{Islamabad,} \mathrm{Pakistan.}{ }^{16} \mathrm{Pediatric}$ Laboratory Medicine, The Hospital for Sick Children, Toronto, Ontario, Canada. ${ }^{17} \mathrm{Department}$ of Medical Physiology, Division of Heart and Lungs, University Medical Center, Utrecht, Utrecht University, Utrecht, Netherlands. ${ }^{18}$ Cardiovascular Department, ASUITS University of Trieste, Trieste, Italy. ${ }^{19}$ Department of Medicine, University of Sao Paulo, Sao Paulo, Brazil. ${ }^{20}$ Knight Cardiovascular Institute, Oregon Health and Science University, Portland, Oregon, USA. ${ }^{21}$ Section of Cardiac Electrophysiology, Division of Cardiology, University of Colorado, Aurora, Colorado, USA. ${ }^{22}$ Division of Cardiology, Sarver Heart Center, University of Arizona, Tucson, Arizona, USA. ${ }^{23}$ McKusick-Nathans Institute of Cenetic Medicine, Johns Hopkins University School of Medicine, Baltimore, Maryland, USA. ${ }^{24}$ Amsterdam University Medical Center, University of Amsterdam, Heart Centre, Department of Clinical and Experimental Cardiology, Amsterdam Cardiovascular Sciences, Amsterdam, Netherlands. ${ }^{25}$ Department of Cardiology, University of Groningen, University Medical Centre Groningen, Groningen, Netherlands. ${ }^{26}$ Robarts Research Institute, Schulich School of Medicine and Dentistry, Western University, London, Ontario, Canada. ${ }^{27}$ Department of Pathology, UCSF, San Francisco, California, USA. ${ }^{28}$ Departments of Clinical Genetics and Clinical and Experimental Medicine, Linköping University, Linköping, Sweden. ${ }^{29}$ Division of Cardiology, Department of Medicine, Dalhousie University, Halifax, Nova Scotia, Canada. ${ }^{30}$ Department of Medicine, Schulich School of Medicine and Dentistry, Western University, London, Ontario, Canada. ${ }^{3}$ Population Health Research Institute, McMaster University, Hamilton, Ontario, Canada. ${ }^{32}$ Amsterdam UMC, University of Amsterdam, Department of Clinical Genetics, Amsterdam, Netherlands. ${ }^{33}$ Department of Cenetics, University Medical Center Utrecht (UMCU), Utrecht, Netherlands. ${ }^{34}$ Division of Cardiology, Department of Medicine, Medical University of South Carolina, Charleston, South Carolina, USA. ${ }^{35}$ Department of Biomedical Engineering, College of Engineering, The Ohio State University, Columbus, Ohio, USA.

\begin{abstract}
Arrhythmogenic cardiomyopathy (ACM) is an inherited arrhythmia syndrome characterized by severe structural and electrical cardiac phenotypes, including myocardial fibrofatty replacement and sudden cardiac death. Clinical management of ACM is largely palliative, owing to an absence of therapies that target its underlying pathophysiology, which stems partially from our limited insight into the condition. Following identification of deceased ACM probands possessing ANK2 rare variants and evidence of ankyrin-B loss of function on cardiac tissue analysis, an ANK2 mouse model was found to develop dramatic structural abnormalities reflective of human ACM, including biventricular dilation, reduced ejection fraction, cardiac fibrosis, and premature death. Desmosomal structure and function appeared preserved in diseased human and murine specimens in the presence of markedly abnormal $\beta$-catenin expression and patterning, leading to identification of a previously unknown interaction between ankyrin-B and $\beta$-catenin. A pharmacological activator of the WNT/ $\beta$-catenin pathway, SB-216763, successfully prevented and partially reversed the murine ACM phenotypes. Our findings introduce what we believe to be a new pathway for ACM, a role of ankyrin-B in cardiac structure and signaling, a molecular link between ankyrin-B and $\beta$-catenin, and evidence for targeted activation of the WNT/ $\beta$-catenin pathway as a potential treatment for this disease.
\end{abstract}

Authorship note: JDR and NM contributed equally to this work Conflict of interest: The authors have declared that no conflict of interest exists. Copyright: (c) 2019, American Society for Clinical Investigation. Submitted: October 15, 2018; Accepted: May 14, 2019; Published: July 2, 2019 Reference information: J Clin Invest. 2019;129(8):3171-3184. https://doi.org/10.1172/JCl125538.

\section{Introduction}

Arrhythmogenic cardiomyopathy (ACM) is an inherited arrhythmia syndrome (prevalence is estimated at 1:2000-5000) associated with fibrofatty replacement of the myocardium, malignant ventricular arrhythmias, and sudden cardiac death (1). The most common sub- 
type involves the right ventricle and is termed arrhythmogenic right ventricular cardiomyopathy (ARVC), though left and biventricular forms are well described (2). Contemporary treatments for ACM, including antiarrhythmic drugs, catheter ablation, and implantable cardioverter defibrillators, attempt to suppress and treat malignant ventricular arrhythmias but fail to address the underlying pathophysiology of this progressive disease (3). The genetic culprits underlying ACM are gradually being identified and have offered critical clues into biological pathways, primarily linked to the desmosome, that may be operative in its pathogenesis (4-9). Although significant progress has been made since the original description of ARVC in 1982 by Marcus and colleagues (10), our understanding of the genetics and pathophysiology governing ACM remains incomplete.

Ankyrins are a family of proteins implicated in the membrane targeting of ion channels and transporters in both excitable and nonexcitable cells. Ankyrin-B (AnkB, encoded by ANK2) targets the $\mathrm{Na} / \mathrm{Ca}$ exchanger and $\mathrm{Na} / \mathrm{K}$ ATPase to the cardiac transverse tubule network, and human ANK2 variants that affect AnkB expression or function are linked with a host of human arrhythmias (11-14). Lack of global AnkB expression is neonatally lethal in mice (15). Thus, the in vivo role of AnkB in cardiac structural regulation is unknown, given the lack of a viable in vivo model.

Here, we report what we believe to be a novel mechanism for human ACM pathogenesis and provide evidence for a potential treatment strategy. Following identification of ANK2 loss-offunction variants in patients with ARVC, development of a mouse model of cardiomyocyte-selective AnkB deletion revealed severe structural changes and premature death. Reflective of human disease, loss of AnkB in mice resulted in chamber remodeling, severe fibrosis, arrhythmia, heart failure, and early mortality. Further, we link AnkB in the hearts of both mice and humans with regulation of $\beta$-catenin, a molecule tightly associated with ACM pathogenesis (7). This pathway is selective, as we observed no effect of AnkB deficiency on the expression or localization of canonical ACMassociated desmosomal proteins. SB-216763, an inhibitor of glyco-

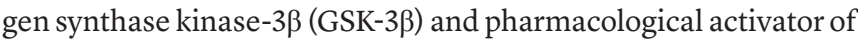
the $\mathrm{WNT} / \beta$-catenin pathway successfully prevented and rescued the ACM phenotype in vivo. Together, we believe our data identify $A N K 2$ as a new, nonconventional ACM disease gene, define new cellular and organ roles for AnkB in cardiomyocytes and cardiac structural regulation and remodeling, and provide evidence to support GSK-3 $\beta$ inhibition as a potential therapy.

\section{Results}

Identification of ARVC on autopsy in a proband with AnkB syndrome. A 52-year-old previously healthy man receiving no medications was referred for a syncopal episode occurring at peak exertion. He had been running approximately 10 kilometers per day at high intensity for 20 years. A baseline ECG showed sinus bradycardia and a normal QT interval (Bazett corrected: $400 \mathrm{~ms}$ ) in the setting of a prolonged QT(U) pattern (Figure 1A). Prior to referral to our clinic, he underwent an invasive electrophysiology study that failed to induce tachycardia. Exercise treadmill testing revealed intermittent pleiomorphic ventricular couplets at peak exertion, and an implantable loop recorder revealed nonsustained polymorphic ventricular tachycardia (VT) during running (Figure 1B). Echocardiography showed moderate biventricular dilation, mildly reduced left ventricular (ejection frac- tion, 45\%-50\%; global hypokinesis) and normal right ventricular systolic function. Cardiac MRI revealed similar biventricular dilation (Figure 1C) and additionally revealed scarring in the right ventricular free wall (Figure 1D) and left ventricular lateral wall (Figure 1E) and the "accordion sign" along the basal right ventricular free wall (Figure $1 \mathrm{C}$ and ref. 16). Clinical genetic testing with pan-arrhythmia and cardiomyopathy panels (79 genes; Supplemental Methods; supplemental material available online with this article; https://doi.org/10.1172/ JCI125538DS1) identified an AnkB-p.Glu1458Gly variant previously linked with AnkB syndrome (11). Aside from 2 TTN missense mutations (p.Asp14812Val and p.Arg22397His) considered benign, no other rare variants were identified. The patient was noncompliant with exercise restriction and beta blockade, declined an implantable cardioverter defibrillator, and subsequently had a fatal cardiac arrest while running at 54 years of age.

Autopsy revealed a markedly enlarged heart (mass: $645 \mathrm{~g}$; normal $342 \pm 40 \mathrm{~g}$ ) and no significant coronary atherosclerosis. On gross examination, the right ventricle was severely dilated, with free wall thinning to less than $0.1 \mathrm{~cm}$ and extensive translucent fat, whereas the left ventricle showed concentric hypertrophy (Figure $1 F)$. Histological analysis revealed that the right ventricular free wall had extensive fat infiltration of the muscle with widespread interstitial fibrosis (Figure $1 \mathrm{G}$ ), whereas the left ventricle had focal fat deposits with interstitial fibrosis (Figure 1H). Analysis performed on right ventricular tissue from the deceased proband reconfirmed the AnkB-p.Glu1458Gly variant as loss of function. In addition to aberrant AnkB localization, we also observed loss of expression of the $\mathrm{Na} / \mathrm{Ca}$ exchanger, an $\mathrm{AnkB}$ partner, at the plasma membrane (Supplemental Figure 1, A and B).

Analysis of the proband's family history revealed a paternal grandfather who died suddenly at 42 years of age, however, the circumstances were unknown and an autopsy was not performed (Supplemental Figure 2A, I-1). The parents and sister of the proband declined an evaluation. Cascade screening identified the AnkB-p.Glu1458Gly variant in 1 of 3 daughters who, in marked contrast to her father, lived a sedentary lifestyle. Her clinical workup at 35 years of age was normal, showing no evidence of AnkB syndrome or ACM (Supplemental Figure 2A, IV-3).

Identification by exome sequencing of a novel ANK2 variant in an ARVC family. On the basis of the initial findings, we expanded our search for ANK2 variants in ACM. Six members from another ARVC family possessing 2 and 3 members with definite and borderline Task Force Criteria-positive diagnoses of genotype-negative ARVC, respectively, underwent exome sequencing (Supplemental Figure 2B). The proband (Supplemental Figure 2B, III-5) was a previously healthy 41-year-old man who died suddenly shortly following intense aerobic exercise and was found to have ARVC on autopsy. Gross examination revealed a moderately dilated right ventricle with fibrofatty infiltration and localized thinning of the anterolateral aspects of the right ventricular free wall, whereas microscopic analysis revealed marked fatty myocardial infiltration, patchy interstitial fibrosis, and myocardial disorganization (Figure 2, A-D). Molecular autopsy with a clinical ARVC genetic panel identified the DSG2-p.Val392Ile (Genome Aggregation Database [gnomAD] allele frequency: 0.26\%) and DSC2-p.Leu732Val (gno$\mathrm{mAD}$ allele frequency: 0.12\%) variants; neither was felt to be sole the culprit, given their gnomAD allele frequencies (17). 
A

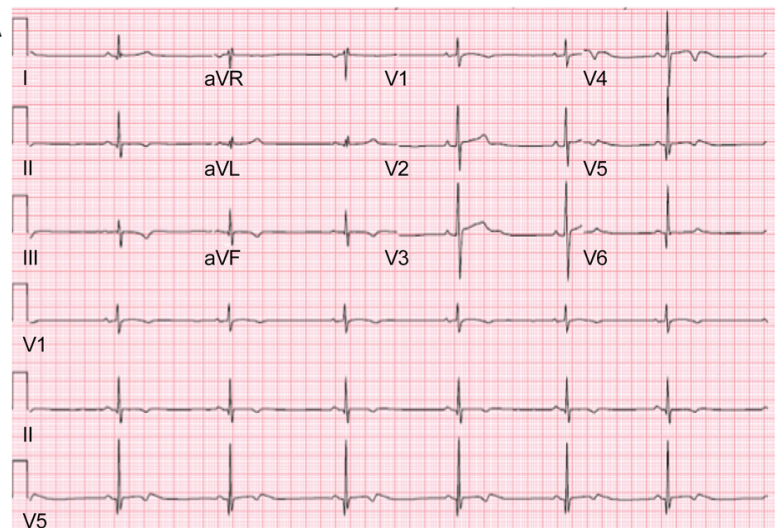

B

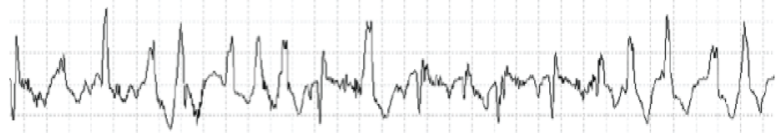

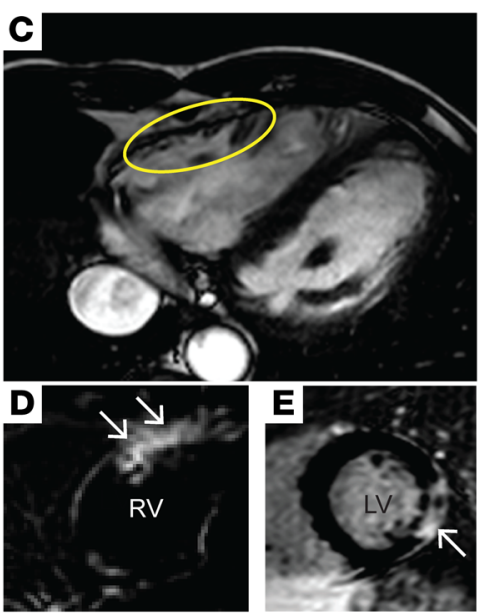

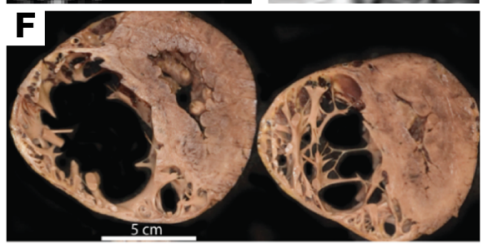

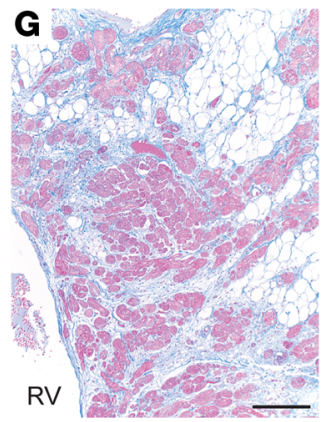

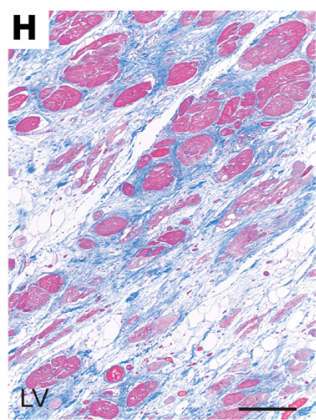

Figure 1. Deceased proband harboring AnkB loss-of-function p.Glu1458Gly variant exhibits arrhythmogenic cardiomyopathy. (A) Surface 12-lead ECG. (B) Loop recording demonstrating nonsustained polymorphic VT. (C) Cardiac magnetic resonance short-axis image revealing moderate biventricular dilation; the yellow ring highlights focal "crinkling" of the subtricuspid region of the right ventricular free wall consistent with the "accordion sign." (D) Delayed enhancement imaging revealed scarring in the right ventricular (RV) free wall in a short-axis view (arrows) and (E) the left ventricular (LV) lateral wall (arrow) in a long-axis view. (F) Mid-transverse section of the autopsied heart revealing severe right ventricular dilation and wall thinning as well as concentric hypertrophy of the left ventricle. Histology of the (G) right ventricle showing severe fibrofatty infiltration of the free wall in association with extensive ventricular interstitial fibrosis and $(\mathbf{H})$ left ventricle showing moderate hypertrophy with focal areas of fibrofatty muscular infiltration and widespread interstitial fibrosis. Scale bars: $160 \mu \mathrm{m}(\mathbf{G})$ and $60 \mu \mathrm{m}(\mathbf{H})$.

Cascade screening revealed that the proband's 83-year-old father (Supplemental Figure 2B, II-12) had a right ventricular free wall aneurysm, mildly reduced right ventricular ejection fraction, and late potentials on signal-averaged ECG (SAECG), consistent with a definite ARVC diagnosis (1 major and 2 minor criteria). Evaluation of an asymptomatic 54-year-old paternal cousin (Supplemental Figure 2B, III-1) revealed a normal 12-lead surface ECG, echocardiogram, and cardiac MRI. On exercise treadmill testing, he suffered a syncopal episode during the recovery period, and a 4-lead surface ECG revealed superiorly directed wide complex tachycardia with a left bundle branch block pattern consistent with VT arising from the right ventricular apex (Figure 2E). These findings were consistent with a borderline diagnosis of ARVC (1 major and 1 minor criteria). Two asymptomatic sisters of the proband (Supplemental Figure 2B, III-2 and III-3) underwent clinical screening at 53 and 57 years of age and were found to have late potentials on SAECG, whereas the remainder of their workup was normal, corresponding to borderline ARVC diagnoses (1 major and 1 minor criteria). The remaining family members were either not accessible or declined evaluation.

Exome sequencing of the 5 aforementioned definite/borderline ARVC family members and the unaffected mother of the proband identified shared variants within 11 genes with gnomAD allele frequencies of less than $0.005 \%$ that segregated with the familial phenotype (Supplemental Table 1). Since late potentials on SAECG may be a nonspecific finding, we performed a similar analysis excluding the sisters of the proband, which yielded shared variants within 23 genes (Supplemental Table 1). Among these genes, only one, ANK2, had previously been implicated in either cardiac physiology or disease, and, correspondingly, the novel AnkB-p.Met1988Thr was considered the top candidate. All variants with allele frequencies of less than $5 \%$ identified from genes previously implicated in cardiomyopathy and arrhythmia and identified in the proband are listed in Supplemental Table 2. The presence or absence of the p.Met1988Thr mutation was confirmed with Sanger sequencing in the affected and unaffected family members, respectively. Notably, the DSG2-p.Val392Ile and DSC2-p.Leu732Val variants also segregated with the ARVC phenotype in the pedigree. Analysis of autopsy tissue from the proband confirmed the AnkB-p.Met1988Thr variant as a lossof-function mutation associated with aberrant AnkB expression, reduced levels of the $\mathrm{Na} / \mathrm{Ca}$ exchanger at the plasma membrane, and abnormal Z-line targeting (Supplemental Figure 1, A and C).

AnkB deficiency causes cardiac structural remodeling and premature death. To assess a potential role of AnkB in postnatal cardiac structure and function, we generated a mouse model of postnatal AnkB deletion in cardiomyocytes. Ank $2^{f / f l}$ mice were generated, validated (described in Methods), and subsequently crossed with $\alpha$-myosin heavy chain-Cre ( $\alpha$ MHC-Cre) mice to selectively eliminate AnkB expression in cardiac myocytes (Supplemental Figure $3 \mathrm{~A}$; $\alpha \mathrm{MHC}-\mathrm{Cre} A n k 2^{f / f l}$, referred to herein as Ank2 cardioselective-KO [Ank2-cKO] mice). Ank2-cKO mice were born at normal Mendelian ratios and, at 8 weeks of age, lacked structural or electrical phenotypes. Immunoblots of cardiac and cerebellar lysates 

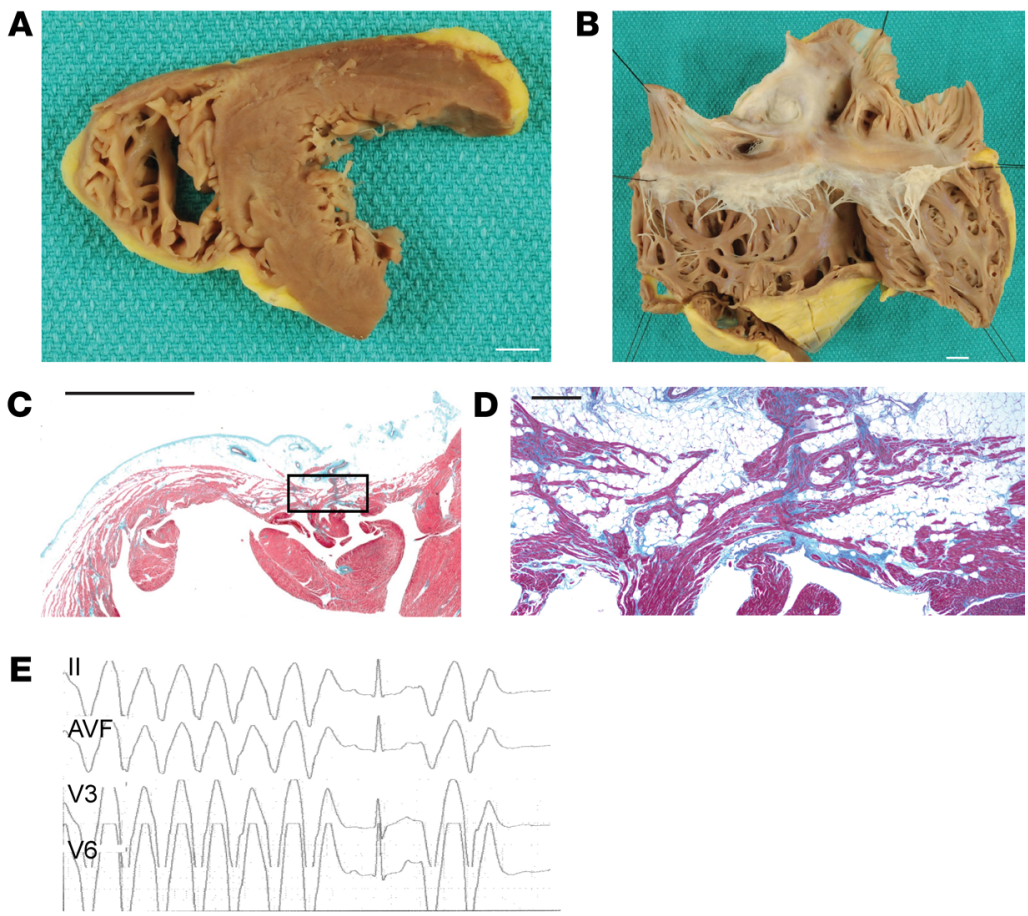

Figure 2. Deceased proband possessing AnkB loss-of-function p.Met1988Thr variant exhibits arrhythmogenic cardiomyopathy. (A) Apical transverse section of the explanted heart of the deceased AnkB-p.Met1988Thr proband revealing fatty infiltration and localized thinning of the anterior and lateral aspects of the right ventricular free wall. Scale bar: 1 $\mathrm{cm}$. (B) Open view of the right ventricle revealing moderate dilation. Scale bar: $1 \mathrm{~cm}$. Histology of the anteroapical right ventricular free wall at (C) $\times 1$ and (D) $\times 40$ original magnification, revealing marked fatty infiltration of the myocardium with patchy interstitial fibrosis and myocardial disorganization. Scale bars: $5 \mathrm{~mm}$ (C) and $300 \mu \mathrm{m}$ (D). (E) Four-lead surface ECG of paternal cousin following a treadmill test, revealing VT. of Ank2-cKO mice confirmed the cardioselective deletion of AnkB (Figure 3A and Supplemental Figure 3, B and E-H). Further, confocal microscopy confirmed the absence of AnkB in cardiomyocytes (Supplemental Figure 3, C and D). At rest, Ank2-cKO animals displayed sinus bradycardia (605 bpm vs. $665 \mathrm{bpm}, P<0.05)$, QT interval prolongation ( $0.020 \mathrm{~s}$ vs. $0.022 \mathrm{~s}, P<0.05)$, and a trend toward QTc prolongation (Supplemental Figure 4, D, G, and H). Following catecholamine challenge ( $2.0 \mathrm{mg} / \mathrm{kg}$ epinephrine), 9 of 10 Ank2-cKO mice had sustained ventricular arrhythmias (defined as lasting longer than 1 second), and 3 died during the stress protocol (Figure 3, D-F, and Supplemental Figure 4, A-C, I, and J).

Unlike normal littermates, Ank2-cKO mice showed severe cardiac remodeling beginning at 10 weeks of age. In Ank2-cKO mice, ventricular dilation associated with increased left ventricular end-systolic and-diastolic diameters was accompanied by reduced left ventricular ejection fraction and fractional shortening, reduced right ventricular fractional shortening, increased heart weight to tibia length ratio, and ultimately premature death (Figure 3, B-D and $\mathrm{G}$, and Supplemental Figure 5). Ank2-cKO hearts showed gross right and left chamber dilation, wall thinning, and widespread cardiac fibrosis consistent with human ACM phenotypes (Figure 3, $\mathrm{H}-\mathrm{K})$. We did not observe significant adipogenesis, however, fatty infiltration is absent from most mouse models of ACM (18-23). Beyond the ventricle, we observed a marked enlargement of the atria compared with control mice (Figure 3, $\mathrm{H}$ and I).

AnkB deficiency alters $\beta$-catenin regulation. ACM is classically linked with molecular remodeling of the cardiac desmosome, a myocyte membrane domain evolved for both myocyte structural and electrical regulation (24). However, we observed no difference in the expression or localization of canonical desmosomal molecules, including desmoplakin, N-cadherin, connexin-43, or plakoglobin, between control and Ank2-cKO hearts (Figure 4, A-D). Further, we observed that expression of desmin and $\alpha$-ac- tinin, both key myocyte cytoskeletal molecules, was unchanged between control and Ank2-cKO hearts (Figure 4, A-D). Protein levels of key intercalated disc molecules, including plakoglobin, plakophilin-2, N-cadherin, desmoplakin, connexin-43, and desmoglein-2, were unchanged between $A n k 2^{f / f l}$ and $A n k 2-c K O$ mice (Supplemental Figure 6, A-F). Disturbances in the cardiac sodium channel have been noted in samples from patients with ACM with diverse genetic causes (25), however, electrophysiological analysis of $\mathrm{Na}_{\mathrm{v}} 1.5$ using whole-cell patch clamping revealed no differences in cardiac sodium channel current $\left(\mathrm{I}_{\mathrm{Na}}\right)$ peak current density, steady-state activation and inactivation kinetics, or recovery from inactivation (Supplemental Figure 7). Unexpectedly, $\beta$-catenin immunolocalization was significantly altered in Ank2-cKO hearts. In addition to decreased expression of $\beta$-catenin at the intercalated disc, we observed significant heterogeneity of the molecule across cardiac sections, as well as notable cytoplasmic puncta and lateralized expression (Figure 4E). In summary, Ank2-cKO animals display severe cardiac remodeling associated with aberrant $\beta$-catenin expression and localization in vivo.

Human hearts from deceased ANK2 ARVC probands display aberrant $\beta$-catenin localization. On the basis of the findings from our mouse model of AnkB deficiency, we investigated the localization and organization of $\beta$-catenin in ventricular sections from nonfailing human heart, as well as in ventricular tissue from the deceased AnkB-p.Glu1458Gly and p.Met1988Thr probands. Consistent with our findings in mice, we did not observe significant differences in desmin expression or localization when comparing sections from nonfailing versus ARVC hearts (Supplemental Figure 8, H-J). Similar to our animal data, we observed abnormal distribution of $\beta$-catenin in the right ventricle of the ANK2 ARVC probands compared with distribution in nonfailing heart sections, including both loss and heterogeneous expression of $\beta$-catenin at the intercalated disc, as well as significant expression of $\beta$-catenin 


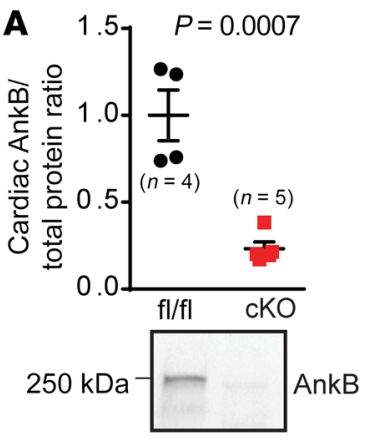

$\mathbf{E}$

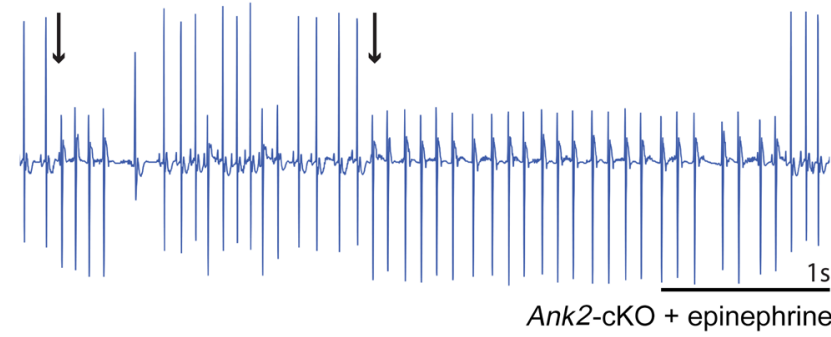

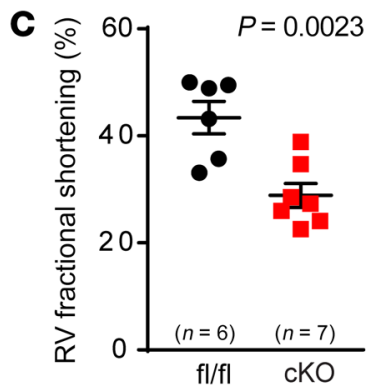

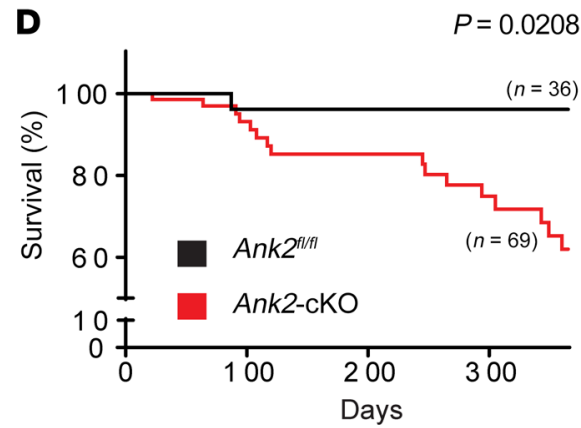

$\mathbf{F}$

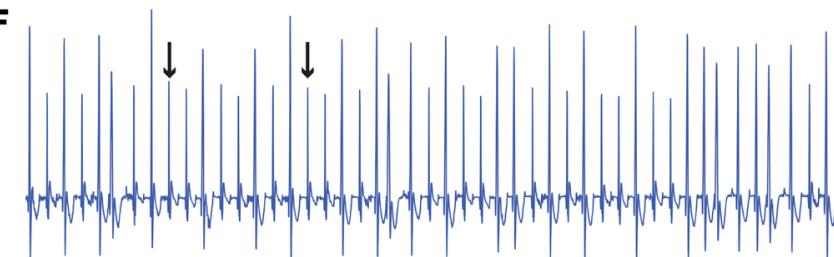

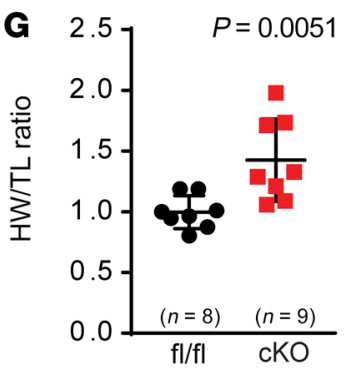
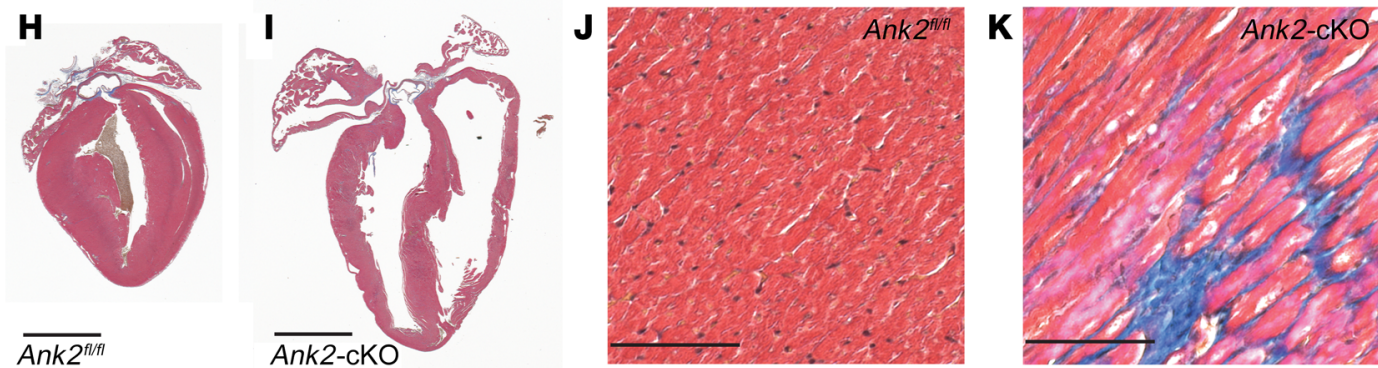

Figure 3. Cardiomyocyte-specific deletion of AnkB results in ventricular remodeling, cardiac arrhythmias, and reduced survival. Immunoblotting for AnkB (A) in heart tissue from Ank2-cKO $(n=4)$ and $A n k 2^{f / f f}(n=5)$ mice (complete immunoblots are shown in Supplemental Figure 3). (B and C) Quantification of (B) left ventricular ejection fraction of $A n k 2^{f \mid / f l}$ mice $(n=4)$ and $A n k 2-c K O$ mice $(n=9)$ and $(C)$ right ventricular fractional shortening in $A n k 2^{f / f l}(n=6)$ and Ank2-cKO ( $n=7)$ mice. (D) Kaplan-Meier survival curves for $A n k 2^{f / f l}(n=36)$ and Ank2-cKO $(n=69)$ mice. (E and F) Representative ECGs for Ank2-cKO mice following injection of $2.0 \mathrm{mg} / \mathrm{kg}$ epinephrine. Arrows denote $(\mathbf{E})$ VT and (F) trigeminy. (C) Heart weight/tibia length $(\mathrm{HW} / \mathrm{TL})$ ratios of $A n k 2^{\mathrm{fl} / f l}(n=8)$ and Ank2-cKO $(n=9)$ mice. ( $(\mathbf{H}$ and $\mathbf{I})$ Representative Masson's trichrome-stained whole slide views of $(\mathbf{H})$ Ank $2^{f / f l}$ and $(\mathbf{I})$ Ank2-cKO and (J) magnified Ank2 $2^{f / f l}$ and (K) Ank2-cKO hearts. Images are representative of 4 hearts from mice of each genotype. Scale bars: $2.5 \mathrm{~mm}(\mathbf{H}$ and $\mathbf{I})$ and $25 \mu \mathrm{m}(\mathbf{J}$ and $\mathbf{K})$. Data represent the mean \pm SEM. Statistical significance for $\mathbf{A}-\mathbf{C}$ and $\mathbf{G}$ was determined using a 2-tailed parametric $t$ test at $95 \% \mathrm{Cl}$ and a log-rank test for $\mathbf{D}$.

in the myocyte cytoplasm (perinuclear; Figure 5, A and B). Loss of normal AnkB or $\beta$-catenin expression and localization was not a generalizable feature of an ARVC heart, as we observed normal localization and expression of both AnkB and $\beta$-catenin in hearts from patients with ACM secondary to either pathogenic PKP2 or $P L N$ variants (Supplemental Figure 9). In summary, our results indicate that loss of AnkB and $\beta$-catenin localization is a feature unique to AnkB-linked ACM and provide a molecular rationale for cardiac remodeling associated with AnkB dysfunction.

$A n k B$ and $\beta$-catenin are molecular partners. On the basis of our cellular data, we tested a potential molecular interaction of AnkB and $\beta$-catenin. Notably, AnkB and $\beta$-catenin interacted in co-IP experiments using detergent-soluble lysates of nonfailing human ventricle (Figure 5C and Supplemental Figure 8). Beyond co-IP experiments, glutathione S-transferase (GST) AnkB membranebinding domain (MBD), but not GST alone, was sufficient to associate with $\beta$-catenin from pull-down experiments using detergent-soluble lysate from mouse ventricle (Figure 5D and Supple- mental Figure 8). A direct interaction of AnkB and $\beta$-catenin was further confirmed by in vitro binding assays, in which GST-AnkB MBD, but not GST, was associated with recombinant ${ }^{35}[\mathrm{~S}]$-Met $\beta$-catenin (Supplemental Figure 8). Our findings illustrate a specific and direct interaction of the AnkB MBD and the C-terminal domain (CTD) of $\beta$-catenin (residues 697-781). It is important to note that, despite abnormal localization in Ank2-cKO hearts, full-length $\beta$-catenin protein levels were unchanged between $A n k 2-c K O$ and $A n k 2^{f / f l}$ control hearts (Figure 5, E and F). In summary, our findings indicate that a direct interaction with AnkB is required for normal $\beta$-catenin localization. These data support what we believe to be a novel role of AnkB in the regulation of cardiac $\beta$-catenin signaling, as well as identify a potential new cellular mechanism underlying human ACM.

GSK-3 $\beta$ inhibitor prevents cardiac remodeling in an ACM mouse model. On the basis of our finding of a direct interaction between AnkB and $\beta$-catenin, coupled with prior studies demonstrating the importance of the WNT/ $\beta$-catenin signaling pathway in ACM, 


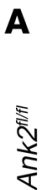
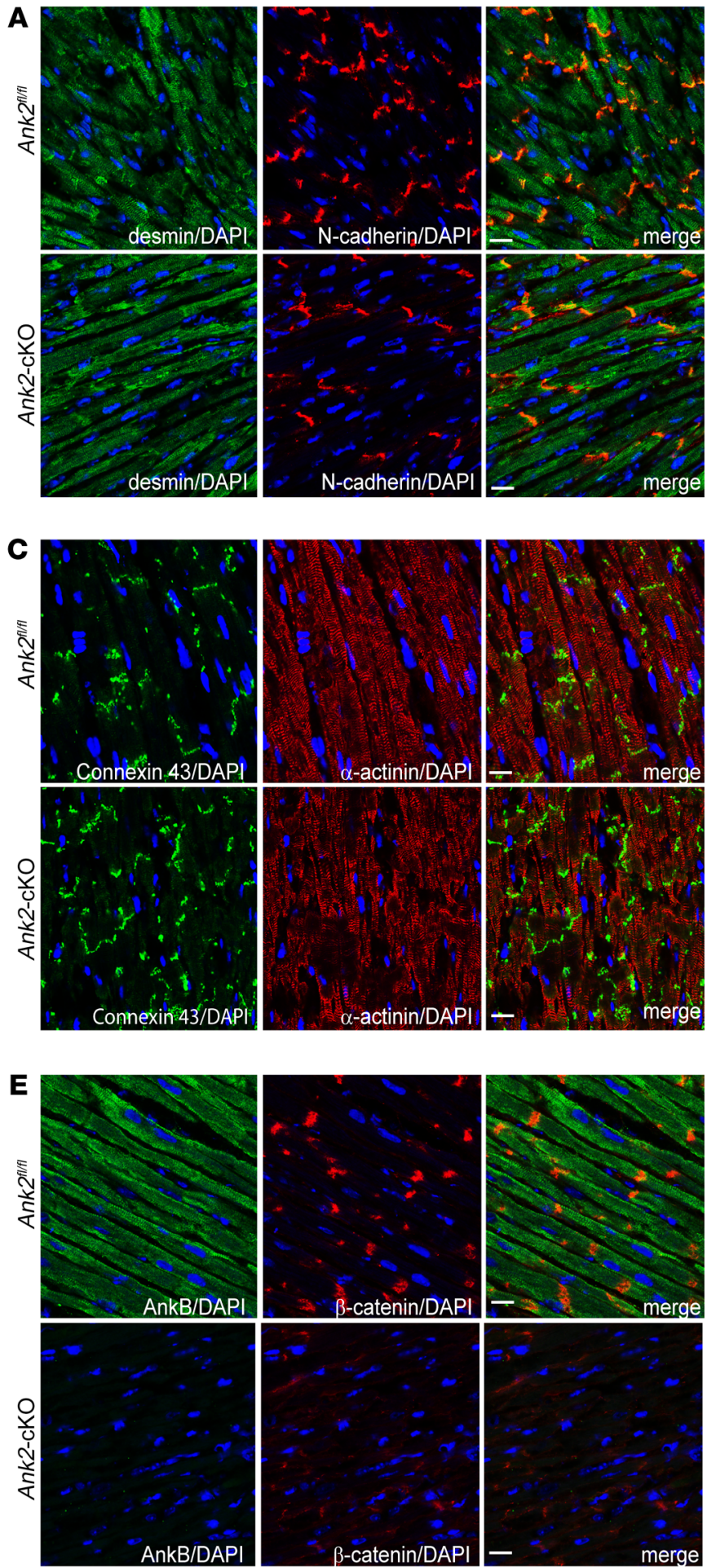

B
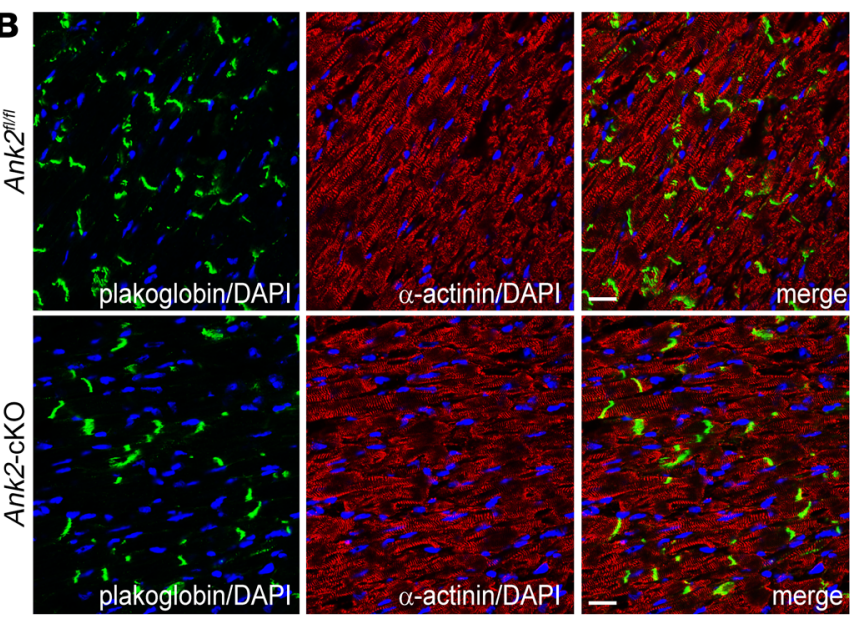

D
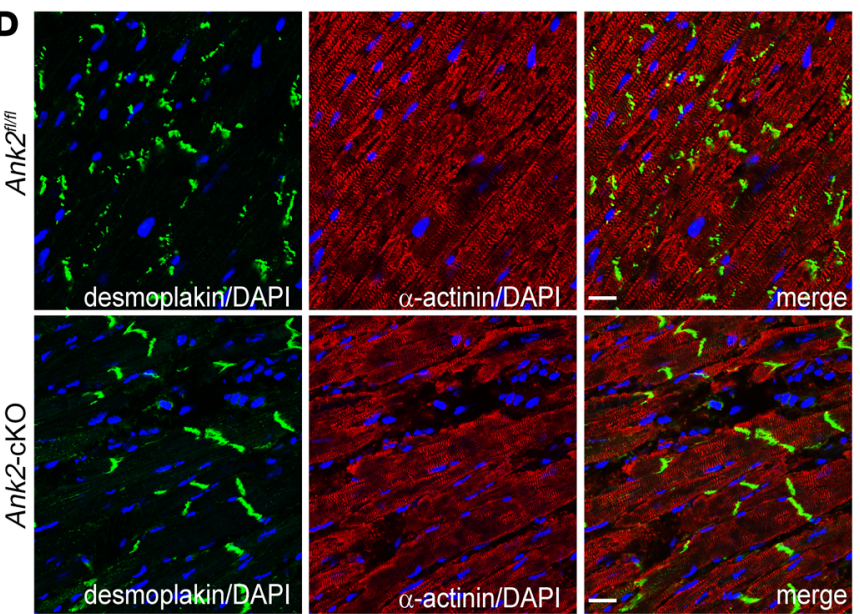

Figure 4. Ank2-cKO murine hearts show misclocalization of $\beta$-catenin. Representative IF images of (A) desmin and N-cadherin, (B) plakoglobin and $\alpha$-actinin, (C) connexin-43 and $\alpha$-actinin, (D) desmoplakin and $\alpha$-actinin, and (E) AnkB and $\beta$-catenin in Ank $2^{f / f f}$ and Ank2-cKO cardiac cryoslices. Staining was completed in tissue from 3 hearts for each stain, per genotype, with 3 images taken per heart. Scale bars: $20 \mu \mathrm{m}$. we tested whether the GSK-3 $\beta$ inhibitor SB-216763, an activator of the $\mathrm{WNT} / \beta$-catenin pathway, is sufficient to prevent cardiac remodeling in Ank2-cKO mice when treatment is initiated at 4 weeks of age $(7,20,26)$. Notably, whereas vehicle-treated Ank2cKO mice showed significant age-dependent cardiac remodeling, an increased heart weight/tibia length ratio, widespread cardiac fibrosis, and a reduced ejection fraction, SB-216763-treated Ank2-cKO mice displayed cardiac phenotypes similar to those of control mice treated with SB-216763 or vehicle at 3 months of age (Figure 6, A, B, and D-K, and Supplemental Figure 10, A-P). Additionally, we observed no significant differences in the number of TUNEL-positive nuclei between $A n k 2^{f / f l}$ and Ank2-cKO mice treated with vehicle or SB-216763 (Supplemental Figure $11, \mathrm{~A}-\mathrm{F})$. Consistent with the activity of GSK3 $\beta$-inhibition, mice injected with SB-216763 showed reduced levels of cardiac phosphorylated $\beta$-catenin ( $\mathrm{p}-\beta$-catenin) (Figure 6C and Supplemental Figure 12, A and B). Both $A n k 2^{f l / f l}$ and Ank2-cKO mice treated with SB-216763 had enhanced diffuse $\beta$-catenin signal, as seen 
A
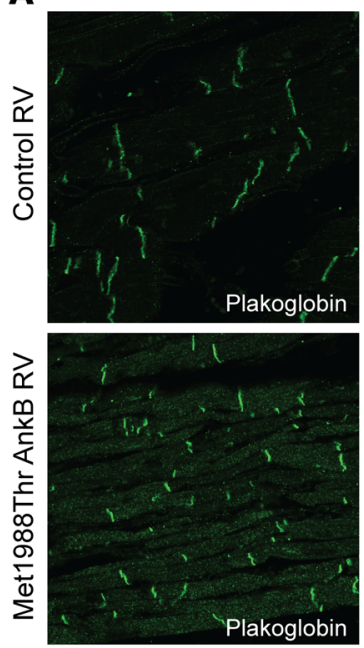

B
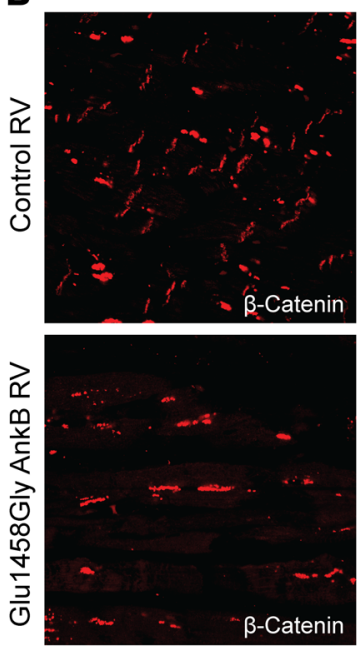
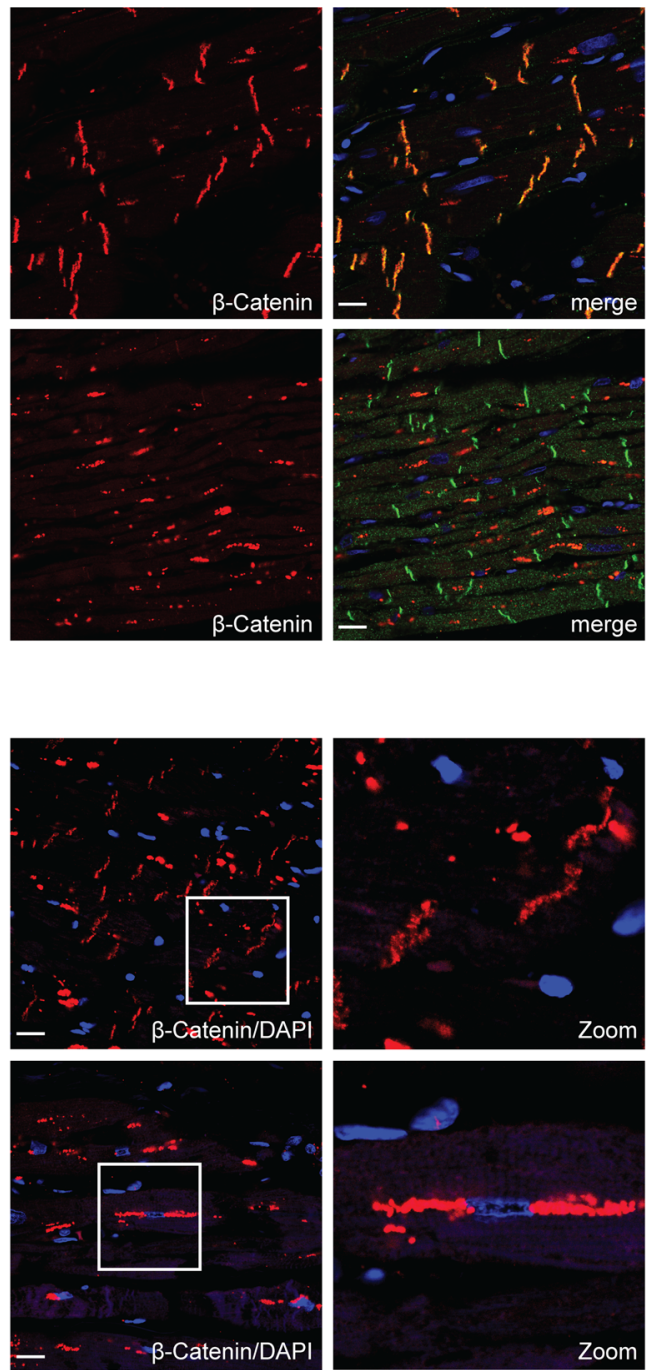

C
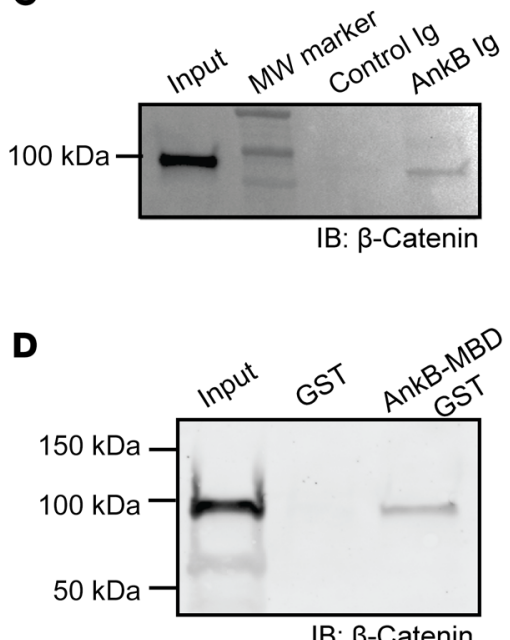

E

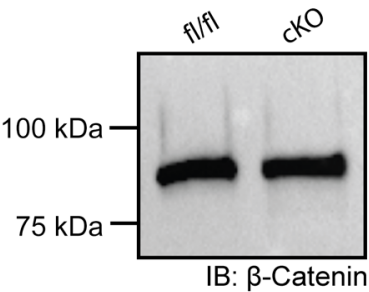

$\mathbf{F}$

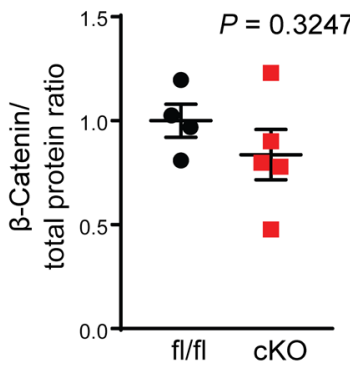

Figure 5. AnkB and $\boldsymbol{\beta}$-catenin are molecular partners. ( $\mathbf{A}$ and $\mathbf{B})$ Representative images of $(\mathbf{A})$ plakoglobin and $\beta$-catenin staining from control and AnkB p.Met1988Thr human right ventricular tissue, and (B) $\beta$-catenin staining of control and AnkB p.Glu1458Gly human right ventricular tissue. Scale bars: 20 $\mu \mathrm{m}$. (C) Co-IP assay of AnkB IgG and $\beta$-catenin in human ventricular lysate. (D) GST-pulldown assay of in vitro-translated AnkB MBD and $\beta$-catenin in mouse cardiac lysate (complete images are shown in Supplemental Figure 8). The binding experiments were replicated 3 times. (E) Representative immunoblot of $\beta$-catenin and (F) quantification of full-length $\beta$-catenin in Ank $2^{f / f f}(n=4)$ and Ank2-cKO $(n=5)$ mouse cardiac lysate (complete images are shown in Supplemental Figure 8). Data represent the mean \pm SEM. Statistical analysis for $\mathbf{F}$ was performed using a 2-tailed parametric $t$ test at $95 \% \mathrm{Cl}$. Binding assays were completed in triplicate.

with immunofluorescence (IF), although in Ank2-cKO mice, failure to completely restore $\beta$-catenin localization to the intercalated disc was observed (Supplemental Figure 10, Q-T). Thus, GSK-3 $\beta$ inhibition is sufficient to prevent cardiac remodeling in the AnkB disease model.

GSK-3 $\beta$ inhibitor rescues cardiac function in an ACM-compromised mouse model. Given the positive findings in the prevention study, we next tested whether GSK-3 $\beta$ inhibition is sufficient to reverse impaired cardiac function in Ank2-cKO mice with established structural changes. As noted previously, 3-month-old Ank2-cKO mice showed marked structural remodeling, including ventricular dilation, fibrosis, and reduced ejection fraction (Figure 3 and Supplemental Figure 5). Although ACM phenotypes progressed in vehicle-treated Ank2-cKO mice, Ank2-cKO mice treated daily with SB-216763 starting at 3 months of age had an improved ejection fraction (Figure 7). In fact, following 4 weeks of daily SB-216763 treatment, Ank2-cKO mice displayed structural phenotypes similar to those of control mice treated with SB-216763 or vehicle $(P=\mathrm{NS})$. Ank2-cKO mice treated with SB-216763 trended toward a lower percentage of fibrosis, as quantified from Masson's trichrome-stained cardiac sections, relative to mice treated with vehicle $(0.99 \%$ vs. $0.31 \%$, $P=0.08$; Supplemental Figure 13, Q-U). Ank2-cKO mice treated with SB-216763 had an increased myocyte cross-sectional area compared with that of DMSO-treated mice $\left(324.5 \mu \mathrm{m}^{2}\right.$ vs. 164.3 $\mu \mathrm{m}^{2}, P<0.05$; Figure 7, F-K). Thus, our findings illustrate that chronic GSK-3 $\beta$ inhibition is sufficient to both prevent and partially reverse ACM phenotypes in the AnkB disease model. 
A

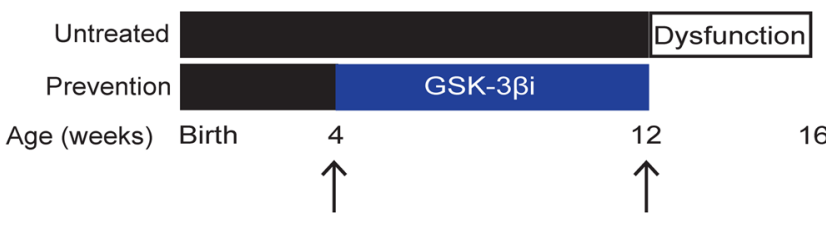

B

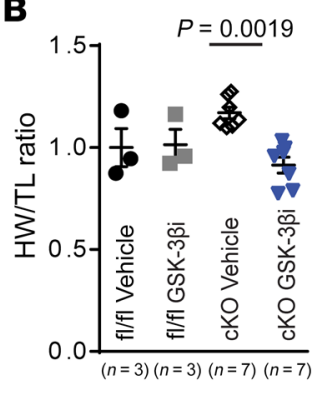

F

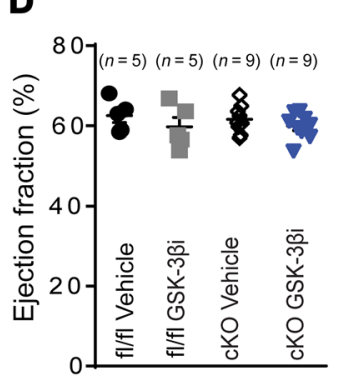

H

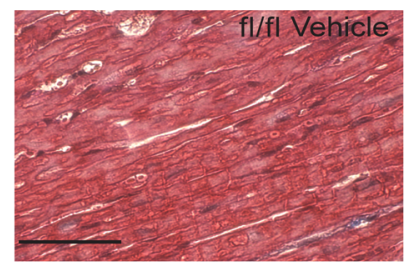

E

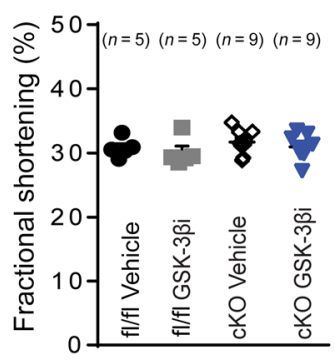

I

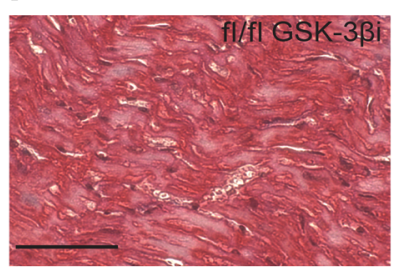

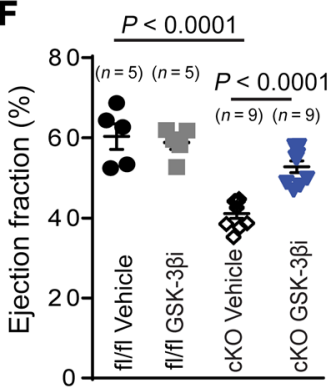

J

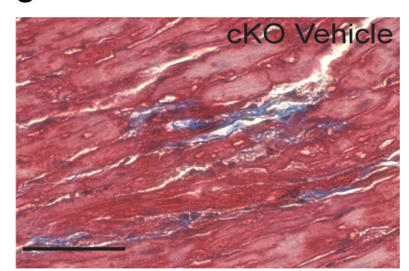

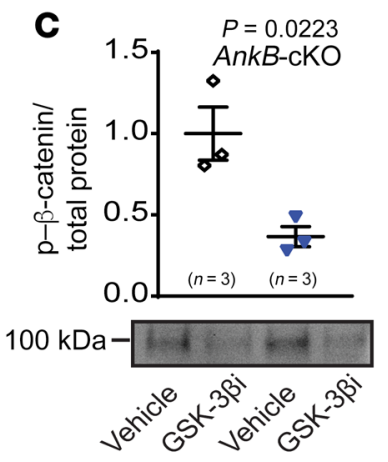

G

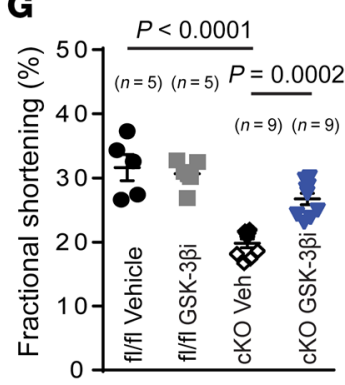

K

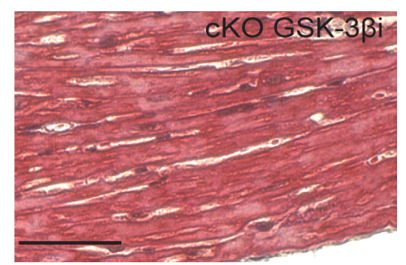

Figure 6. GSK3- $\beta$ inhibition is sufficient to prevent cardiac remodeling associated with cardiac deletion of AnkB. (A) Diagrammatic representation of GSK3$\beta i$ prevention study in $A n k 2^{f / f l}$ and $A n k 2-c K O$ mice. Echocardiograms were performed at the time points indicated by arrows. (B) Heart weight/tibia length ratios. $n=3$. (C) Levels of $p-\beta$-catenin by IB of $A n k 2$-cKO cardiac tissue lysates. $n=3$. (D and $\mathbf{E}$ ) Ejection fraction and fractional shortening at baseline. Ejection fraction (F) and fractional shortening (G) after 8 weeks of drug therapy in vehicle- and GSK3- $\beta$ i-treated Ank2 $2^{f / f l}$ and Ank2-cKO mice in the prevention study. $n$ $=5$ (D and $\mathbf{E})$ and $n=9$ (F and $\mathbf{C})$. (H) Representative Masson's trichrome-stained heart sections from vehicle-treated Ank2 $2^{f / f l}$ mice. (I) Ank $2^{f / f f l}$ CSK- $\beta \mathbf{i}-$ treated, (J) Ank2-cKO vehicle-treated, and (K) Ank2-cKO CSK3- $\beta$ i-treated mice after 8 weeks of drug therapy in the prevention study. Scale bars: $25 \mu \mathrm{m}$ (I-K). Images are representative of 2 hearts from mice of each genotype and treatment condition. Data represent the mean \pm SEM. Statistical analysis for $\mathbf{C}$ was done with a 2-tailed parametric $t$ test at $95 \% \mathrm{Cl}$. Statistical analysis for B and D-G was performed with a 2-way ANOVA followed by Tukey's post hoc test.

Identification of ANK2 rare variants in a genotype-negative ARVC cohort. We subsequently performed ANK2 genetic screening in a multicenter cohort of 207 unrelated genotype-negative, Task Force Criteria-positive (definite diagnosis) ARVC probands to further evaluate the contribution of ANK2 to ACM. The mean age of the study participants at presentation was 39.2 years (SD: 14.3 ), $59.4 \%$ were male, $98.6 \%$ were white, and the mean number of major and minor Task Force Criteria was 1.9 (SD: 0.8) and 1.6 (SD: 1.1), respectively. The remaining clinical characteristics are listed in Supplemental Table 3. A total of 14 ANK2 rare variants (defined as minor allele frequency $<0.1 \%$ ) were identified among 13 individuals in the study cohort, corresponding to a cumulative carrier frequency of $6.8 \%$ (Supplemental Table 4). The allele frequencies, domain locations, and results of in silico analyses of the ANK2 variants are provided in Supplemental Table 5.

The cumulative frequency of the 14 rare variants identified in our ARVC cohort was increased relative to their cumulative frequency in gnomAD $(6.8 \%$ vs. $0.7 \%, P<0.001)$. Within gno-
$\mathrm{mAD}$, a total of 1895 missense, small insertion-deletion, or radical (defined as stop-gain, stop-loss, frameshift, or canonical splice site) ANK2 genetic variants with allele frequencies of less than $0.1 \%$ are reported with an overall cumulative frequency of $9.0 \%$ (17). We observed no difference when the cumulative frequency of all missense, small insertion-deletion, or radical ANK2 rare variants was compared between the 2 cohorts ( $6.8 \%$ vs. $9 \%, P=0.33)$.

Clinical features of probands possessing rare ANK2 variants. Clinical features of the 13 ARVC probands harboring ANK2 variants from the multicenter ARVC cohort are summarized in Supplemental Table 6. The age of the probands at presentation ranged from 12 to 59 years, 9 of $13(69 \%)$ were male, and all were white. One proband died suddenly and had ARVC confirmed on autopsy (AnkB-p. Arg2069His), one had a successfully resuscitated, aborted cardiac arrest, and an additional nine probands had documented sustained monomorphic VT. Analysis of right ventricular free wall tissue from the deceased AnkB-p.Arg2069His proband revealed aberrant AnkB subcellular localization (Supplemental Figure 14). Beyond ventricular arrhythmias, 2 individuals were diagnosed with typical 
A
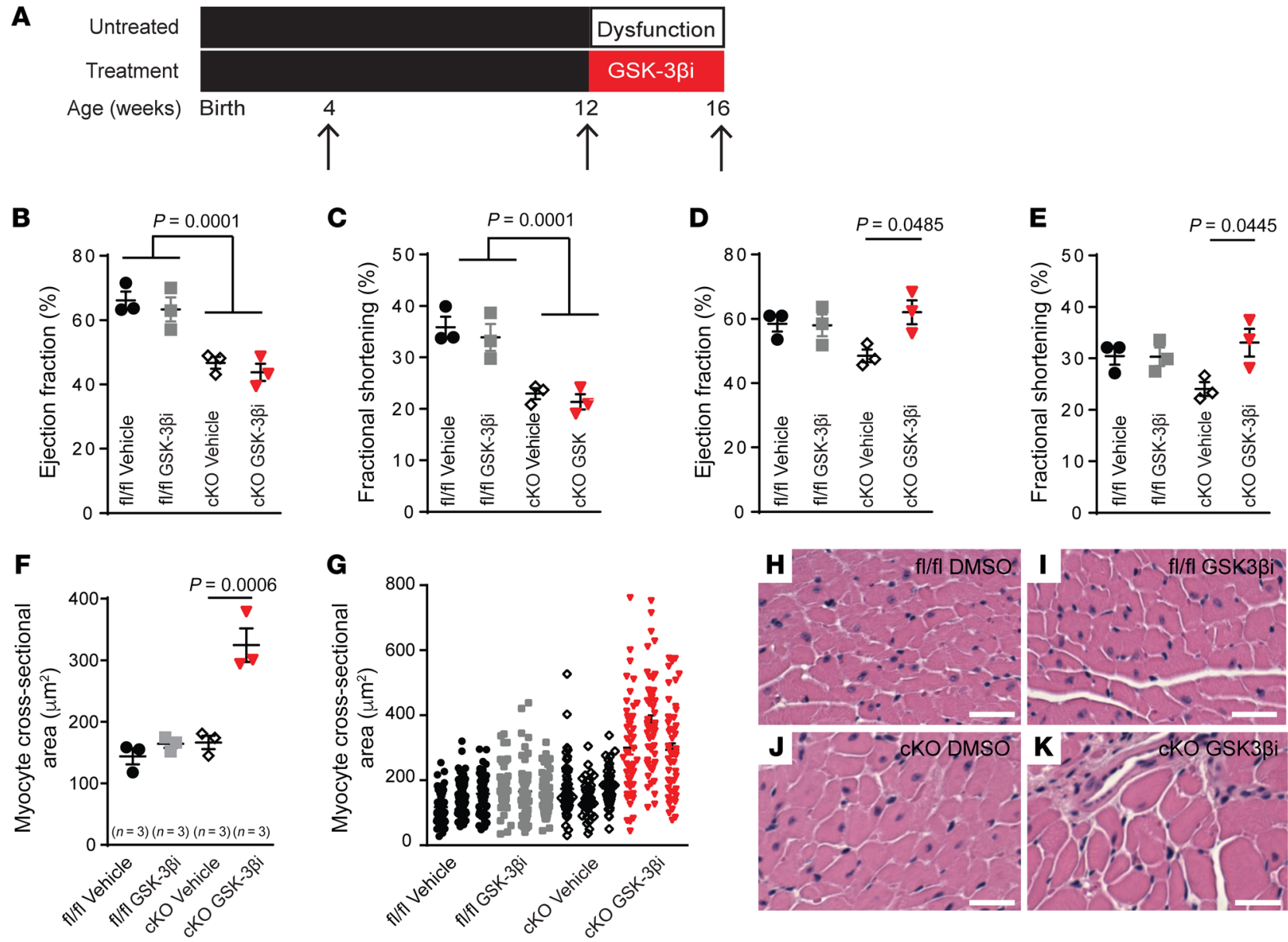

Figure 7. GSK3- $\beta$ inhibition is sufficient to reverse cardiac remodeling associated with cardiac deletion of AnkB. (A) Diagrammatic representation of the GSK3- $\beta$ i rescue study involving $A n k 2^{f / f f}$ and $A n k 2$-cKO mice. Echocardiograms were performed at the time points indicated by arrows. Ejection fraction (B) and fractional shortening (C) at 12 weeks of age and ejection fraction (D) and fractional shortening (E) after 4 weeks of drug therapy in vehicle- and GSK3- $\beta$ i-treated $A n k 2^{f / / f l}$ and Ank2-cKO mice. $n=3(\mathbf{B}-\mathbf{E})$. (F and $\left.\mathbf{G}\right)$ Myocyte cross-sectional area in $A n k 2^{f^{f / f l}}$ ( $n=3$ for each treatment) and $\operatorname{Ank2-cKO~(~} n=3$ for each treatment) mice following treatment with vehicle or GSK3- $\beta$ i. (H-K) Representative cross-sectional images of $A n k 2^{f / f l}$ and Ank2-cKO hearts following vehicle and GSK3- $\beta$ i therapy. Images are representative of 3 hearts from mice of each genotype and treatment condition. Scale bars: $25 \mu \mathrm{m}$. Data represent the mean \pm SEM. Statistical analysis for B-F was performed with a 2-way ANOVA followed by Tukey's post hoc test.

atrial flutter and had successful cavotricuspid isthmus ablations. None of the 13 probands was documented to have QT prolongation.

Although each individual was classified as genotype negative, 3 had rare variants in cardiomyopathy genes (see Supplemental Table 6 for allele frequencies and in silico analyses). A desmosomal variant of unknown significance (DSP-p.Asn593Ser; gnomAD allele frequency $=0.05 \%$ ) was observed in the proband possessing the AnkB-p.Ile964Val variant. The MYH6 gene has not been implicated in ARVC, and a pathogenic SCN5A-p.Phe861TrpfsTer90 mutation was identified in the proband possessing the AnkB-p.Thr3744Asn variant.

\section{Discussion}

Here, we provide the first evidence to our knowledge implicating AnkB loss of function in structural heart disease and ACM; identify a mechanistic rationale involving $\beta$-catenin signaling through a previously unknown AnkB interaction that appears independent of the desmosome; and demonstrate that GSK-3 $\beta$ inhibition is able to both prevent and rescue the phenotype.
Our study was initiated following autopsy findings of severe ARVC in a sudden death victim who had a premortem clinical phenotype of AnkB syndrome secondary to the AnkB-p.Glu1458Gly variant. The ANK2 variant was considered an attractive candidate, given its established importance in cardiac disease, which has included robust evidence of genotype-phenotype segregation in large families and detailed in vitro and in vivo analyses of the underlying pathophysiology of ANK2 $(11,27)$. The significance of our initial finding was bolstered following identification of a novel AnkB-p.Met1988Thr mutation segregating with an arrhythmic phenotype in a separate ARVC kindred using exploratory exome sequencing. Analyses of the hearts from the deceased probands of both families were consistent with AnkB loss of function. Notably, cardiac desmosomal structure and function appeared intact in both cases, suggesting a potential novel mechanistic pathway for ARVC development independent of the desmosome.

The importance of AnkB loss of function in relation to structural heart disease was subsequently supported by a cardioselective KO Ank2 mouse model that developed dramatic structural 
abnormalities by 10 weeks of age, including marked biventricular dilation, fibrosis, and reduced ejection fraction, despite apparently preserved desmosomal structure and function. Fat deposition is a hallmark of ACM in humans (28), however, its presence in murine models of disease is variable. Numerous genetic models of ACM, including those involving plakoglobin, plakophilin-2, and desmoglein-2, have failed to demonstrate adipocyte infiltration (18-23). Thus, the absence of fatty infiltration in Ank2-cKO hearts was not unexpected. The precise mechanisms underlying reduced cardiac function in the Ank2-cKO mice remain to be determined, and we acknowledge that future experiments will be necessary to dissect the culprit molecular and cellular pathways and to further our understanding of the therapeutic effect of SB-216763.

Our findings of a direct interaction between AnkB and $\beta$-catenin and heterogeneous expression patterns of $\beta$-catenin observed in the hearts of the Ank2-cKO mice and deceased human probands provide support for altered $\beta$-catenin homeostasis as a mechanism for the observed structural remodeling. These data are further supported by our findings that GSK-3 $\beta$ inhibition is effective at both preventing and reversing the cardiac structural and tissue remodeling in Ank2-cKO animals. AnkB in this novel role may also impact $\beta$-catenin indirectly by altering the phosphorylation status of $\beta$-catenin through AnkB interactions with protein phosphatase $2 \mathrm{~A}(29,30)$. Although we have hypothesized that altered $\beta$-catenin may serve to promote cardiomyopathy, other molecular factors may support structural remodeling in the Ank2-cKO mouse model. Certainly, chronic altered calcium handling observed in AnkB disease may alter both transcriptional and structural programming in the heart. Further, ankyrins are associated with a host of structural and signaling proteins in heart and other excitable tissues that may affect disease progression. Importantly, we hypothesize that altered $\beta$-catenin localization produces a phenotype unique from that of simple $\beta$-catenin deficiency. Notably, a model of inducible $\beta$-catenin deletion in the adult mouse does not display cardiac phenotypes, although the model is based on phenotypes in an acute (4-week-KO) and unstressed animal (31).

The decision to trial SB-216763, a GSK-3 $\beta$ inhibitor and pharmacological activator of the $\mathrm{WNT} / \beta$-catenin pathway, as a treatment was driven by prior studies involving zebrafish and murine models of ACM, coupled with the findings in our current study (20, 26). As a preventive therapy administered from 4 weeks of age, a time point that precedes the development of structural changes, its efficacy was remarkable, with the cardiac phenotype of Ank2cKO mice at 3 months of age being indistinguishable from that of their healthy Ank2 $2^{f / f l}$ counterparts. Perhaps even more striking was the ability to partially reverse the ACM phenotype when administration was initiated after marked structural changes had already developed. Despite the presence of severe biventricular dilation, fibrosis, and reduced ejection fraction in 3-month-old mice, following 1 month of SB-216763 administration, the cardiac phenotype returned to near normal. Given the increased myocyte cross-sectional area observed following administration of SB-216763, we hypothesize that the improved ventricular function was mediated through hypertrophy and increased contractility of surviving myocytes. To our knowledge, this is the first study to show the ability of SB-216763 to ameliorate an ACM phenotype in mice and is a critical finding, given that many patients with
ACM initially present with marked structural changes. Although implantable cardioverter defibrillators can be life-saving and antiarrhythmic drugs and catheter ablation may be effective in suppressing malignant ventricular arrhythmias, there are no available therapies that directly target the underlying pathophysiology of ACM (3). The ability to halt and reverse progression of the disease through targeted medical therapy would be a critical advance for patients with ACM. It should be noted that SB-216763, as a compound that upregulates WNT signaling, has a theoretical risk of oncogenesis, though, to our knowledge, no oncogenic signals have been noted in murine or zebrafish models to date $(20,26)$. Nonetheless, it may be necessary to pursue alternative therapeutic compounds and strategies to target this pathway.

Although the cumulative allele frequency of the 14 variants in our multicenter ARVC cohort exceeded that in gnomAD, the cumulative frequency of all ANK2 rare variants anticipated to be functional did not differ between the 2 cohorts. It is conceivable that the $A N K 2$ variants identified in our cohort are unique in their ability to predispose patients to ARVC, however, the lack of enrichment observed when all ANK2 variants were considered may also be secondary to inadequate statistical power, provided a true association exists, or may suggest that disease-relevant ANK2 variants require additional genetic and/or environmental insults, such as intense endurance exercise, to cause an ACM phenotype (32). This concept is further supported by the allele frequencies of many ANK2 variants identified being too common to be causative in isolation, coupled with the frequent lack of family history in cases. Although potentially viewed as a limitation, this phenomenon is probably operative in the majority of genetic ACM subtypes, in which the importance of polygenic drivers and gene dosage is becoming increasingly apparent (33-36). Indeed, the notion of ACM being a polygenic disease dependent on multiple "hits" will almost certainly be the rule rather than the exception for the vast majority of remaining ACM genotype-negative cases that are overwhelmingly sporadic (37).

Limitations. As noted above, although the clinical and pathological phenotypes in this study are striking and decisive, future work will be necessary to clarify the role of genetic and environmental modifiers contributing to an ACM phenotype in the setting of a pathogenic $A N K 2$ variant. Next-generation sequencing techniques have provided an abundance of genetic data, however, we still lack a detailed understanding of vast noncoding regions of the genome that will influence disease phenotypes. Although AnkB dysfunction - whether via promoter variants that reduce expression (14), lossof-function variants that either disrupt tissue expression or cellular function $(12,27,38)$, or chromosomal reorganization that affects AnkB expression and activity (39) - is linked with human disease phenotypes, the penetrance and severity of cardiac phenotypes is often variable. Further detailed evaluation of individuals in this study, as well as others, will likely elucidate more complete genetic and environmental profiles underlying the disease and disease penetrance. Future studies utilizing AnkB-p.Glu1458Gly- or p.Met1988Thr-knockin models may also provide additional mechanistic data underlying ACM pathogenesis due to AnkB loss of function.

Conclusions. We believe our study identifies a novel genetic culprit and molecular pathway for ACM and provides the first evidence to implicate AnkB in the development of structural heart 
disease. Our findings provide insight into the underlying mechanism, which appears to be mediated through WNT/ $\beta$-catenin signaling secondary to a novel AnkB- $\beta$-catenin interaction that is independent of the cardiac desmosome. Last, we provide evidence to support GSK-3 inhibition as a therapy capable of both prevention and rescue of the ANK2-mediated ACM phenotype.

\section{Methods}

Study population. The initial proband for this study underwent clinical evaluation, management, and subsequent autopsy at the UCSF Medical Center. Clinical and genetic cascade screening was performed in consenting first-degree family members.

Whole-exome sequencing in a multigenerational ARVC kindred. Selected affected and unaffected members of a multigenerational genotype-negative ARVC kindred underwent whole-exome sequencing for gene discovery. Whole-exome sequencing was performed for 5 definite or borderline (as defined by the Task Force Criteria) affected individuals and 1 unaffected family member; sequencing and bioinformatic methods are provided in the Supplemental Methods. Shared variants with allele frequencies of less than $0.005 \%$, as found in gnomAD, were filtered to generate a candidate gene list. The top candidate was selected on the basis of biological plausibility, and its segregation among family members was then confirmed with Sanger sequencing. All variants with allele frequencies of less than $5 \%$ identified from genes previously implicated in cardiomyopathy and arrhythmia identified in the proband are provided in Supplemental Table 2.

Ank2-cKO mice. Ank2-cKO mice were generated by the introduction of LoxP sites flanking exon 24 of the ANK2 gene. This strategy results in the deletion of $73 \mathrm{bp}$ of coding sequence: the splicing of exon 23 to exon 25 leads to a frameshift resulting in a premature stop codon in exon 25. Mice were crossed to generate pure lines of floxed mice devoid of the neomycin cassette. Mice were screened by PCR and Southern blot analysis (genOway). Mice were backcrossed onto a C57/ BL6 background more than 5 times. Animals were crossed with mice expressing the Cre recombinase under the cardiac $\alpha \mathrm{MHC}$, resulting in specific loss of AnkB in adult cardiac myocytes.

Animals. All mice used were male and female littermates between 4 and 16 weeks of age, with the exception of those allowed to live up to 1 year as part of the survival studies. The mice were housed in the same facility, consumed the same diet, provided water ad libitum, and kept on identical 12-hour light/12-hour dark cycles. Supplemental Table 7 delineates the sex and age of all mice used in physiological experiments.

Immunoblots. Murine tissue was harvested and immediately placed into cold NP-40 substitute homogenization buffer. Following protein quantification, lysates were boiled for 5 minutes at $95^{\circ} \mathrm{C}$ in 20:1 Laemmli sample buffer (Bio-Rad) and $\beta$-mercaptoethanol and electrophoresed on a Mini-PROTEAN Tetra Cell (Bio-Rad) on a $4 \%-15 \%$ precast gel (Bio-Rad). Following transfer to nitrocellulose, the membranes were blocked for 30 minutes using either 3\% BSA or $5 \%$ blocking buffer (Bio-Rad), depending on the protein of interest. Next, the membranes were incubated with a primary antibody overnight and then washed and incubated with a secondary antibody for 2 hours. Densitometric analysis was performed using ImageJ software $(\mathrm{NIH})$. For all experiments, protein values were normalized against an internal loading control (GAPDH) or against total protein loading quantification via Ponceau staining.
Immunostaining. For cryoslices and paraffin-embedded tissue, heart sections from $A n k 2^{f / f l}, A n k 2-c K O$, and human cardiac tissue were cut at $5-\mu \mathrm{m}$ thickness, blocked in blocking solution (3\% fish gelatin, $0.1 \%$ DMSO, $0.075 \%$ Triton $\mathrm{X}-100$ ), and then incubated overnight at $4^{\circ} \mathrm{C}$. Paraffin sections were deparaffinized using xylenes and ethanol, and antigens were retrieved using pepsin solution for 15 minutes at $37^{\circ} \mathrm{C}$. After secondary antibody treatment, the sections were extensively washed and covered with VECTASHIELD imaging medium (Vector Laboratories), and coverslips (no. 1) were applied. Images were acquired with a confocal microscope (510 Meta, Carl Zeiss) with a $40 \times$ water 1.30 NA lens (pinhole equals 1.0 airy disc; Carl Zeiss) and imaging software (release version 4.0 SP1; Carl Zeiss). The images were collected using similar confocal protocols at room temperature.

Antibodies. The antibodies used included the following: AnkB (1:2000 immunoblotting [IB], 1:100 IF, custom-made by Covance); $\beta$-catenin (1:1000 IB, 1:100 IF; BD Biosciences, 610153); $\alpha$-actinin (1:400 IF; MilliporeSigma); desmoplakin (1:1000 IB, 1:100 IF; Abcam, ab16434); N-cadherin (1:1000 IB, 1:100 IF; Thermo Fisher Scientific, 33-3900); desmin (1:200 IF; MilliporeSigma, D8281); plakoglobin (1:1000 IB, 1:100 IF; Abcam, 15153); desmoglein-2 (1:1000, IB; Invitrogen, Thermo Fisher Scientific, 69369); connexin-43 (1:1000, IB, 1:100 IF; Thermo Fisher Scientific, 71-0700); p- $\beta$-catenin (1:1000 IB; Cell Signaling Technology, 9561); GAPDH (1:10,000 IB; Fitzgerald); and $\beta$ II spectrin (1:100 IF; Covance). Secondary antibodies for IB included donkey anti-mouse HRP and donkey anti-rabbit HRP (The Jackson Laboratory) as well as goat anti-mouse and goat anti-rabbit StarBright 700 Fluorescent Secondary Antibodies (Thermo Fisher Scientific). Secondary antibodies for IF included anti-rabbit and antimouse Igs conjugated to Alexa Fluor 488 or Alexa Fluor 568 (1:400; Invitrogen, Thermo Fisher Scientific). BD Biosciences produced the $\beta$-catenin antibody against the 571-781 amino acids of the murine $\beta$-catenin protein, which corresponds to the 11th and 12th armadillo repeats (ARMs) and the C-terminal portion of the protein.

$\beta$-Catenin antibody validation. HEK293 cell lysates were gathered, subjected to SDS-PAGE, transferred to a nitrocellulose membrane, and probed with $\beta$-catenin antibody.

Echocardiography. Transthoracic echocardiography was performed using the Vevo 2100 (VisualSonics). The mice were anesthetized using $2.0 \%$ isoflurane in $95 \% \mathrm{O}_{2}$ and $5 \% \mathrm{CO}_{2}$ at a rate of approximately $0.8 \mathrm{~L} / \mathrm{min}$. Anesthesia was maintained by administration of oxygen and approximately $1 \%$ isoflurane. Electrode gel was placed on the ECG sensors of the heated platform, and the mouse was placed supine onto the platform to monitor electrical activity of the heart. A temperature probe was inserted into the rectum of the mouse to monitor its core temperature of approximately $37^{\circ} \mathrm{C}$. The MS-400 transducer was used to collect the contractile parameters and chamber dimensions of the left ventricle using M-mode in the short axis. For assessment of the right ventricle, fractional shortening and chamber dimensions were evaluated in a modified long axis of the heart (40). 2D images were obtained for confirmation of proper orientation.

Mortality studies. Survival of Ank2-cKO mice $(n=69)$ mice was compared with that of $A n k 2^{f / f l}$ animals $(n=36)$. Mice in this study were followed for 12 months or until death, whichever came first. Mice were allowed to run freely in cages but were not subjected to secondary exercise or environmental protocols. 
Tissue pathology for hearts from mice and deceased human probands. Whole hearts were excised from 2- to 3-month-old male Ank $2^{f / f l}$ and Ank2-cKO mice, whereas the hearts from the deceased human probands who had the AnkB-p.Glu1458Gly and -p.Met1988Thr mutations were obtained at the time of autopsy. Hearts were rinsed in PBS, fixed in $10 \%$ formalin solution, and then paraffin embedded. Hearts were sliced at 5- $\mu \mathrm{m}$ thickness and stained with Masson's trichrome at The Ohio State University's murine pathology core laboratory. Whole heart images were obtained using the PathScan Enabler IV slide imager (Meyer Instruments), and magnified images were acquired with a Thermo Fisher Scientific EVOS microscope. Human samples for Supplemental Figure 8 were derived from 4 ACM probands (with pathogenic variants in either PKP2 or PLN) and 5 control hearts. None of the 4 ACM patients had the aberrancies that were observed in the ANK2 variant carriers. These human cardiac specimens were obtained from the cardiac tissue bank of the Department of Pathology at the University Medical Center (Utrecht, Netherlands). The scientific advisory board of the University Medical Center Utrecht (Biobank) approved this component of the study.

Myocyte cross-sectional area analysis. Images of cross-sectional myocytes from H\&E-stained murine cardiac sections were obtained using a Thermo Fisher Scientific EVOS microscope. ImageJ software $(\mathrm{NIH})$ was used to determine the cross-sectional area of 50 myocytes per heart section.

TUNEL analysis. TUNEL staining was performed using a Fluorescein In Situ Cell Death Detection Kit (Roche, 11684795910), and TUNEL-stained cardiac sections were imaged using an EVOS microscope. TUNEL-stained images were analyzed using Image (NIH) with "color threshold" and "analyze particle" functions. Only particles of greater than 150 pixels in size were counted as nuclei. The numbers of TUNEL-positive nuclei were normalized to total nuclei (DAPI).

Electrophysiological studies of $\mathrm{Na}_{v} 1.5$. Sodium current recordings were conducted in a low-sodium extracellular solution containing $10 \mathrm{mM} \mathrm{NaCl}, 1 \mathrm{mM} \mathrm{MgCl} 2,1.8 \mathrm{mM} \mathrm{CaCl} 2,0.1 \mathrm{mM} \mathrm{CdCl} 2,20 \mathrm{mM}$ HEPES, $127.5 \mathrm{mM} \mathrm{CsCl}$, and $11 \mathrm{mM}$ glucose. The pipette solution contained $5 \mathrm{mM} \mathrm{NaCl}, 135 \mathrm{mM} \mathrm{CsF}, 10 \mathrm{mM}$ EGTA, $5 \mathrm{mM}$ MgATP, and $5 \mathrm{mM}$ HEPES. To characterize the voltage dependence of the peak $\mathrm{I}_{\mathrm{Na}}$, single cells were held at $-120 \mathrm{mV}$, and $200 \mathrm{msec}$ voltage steps were applied from -100 to $+10 \mathrm{mV}$ in $5-\mathrm{mV}$ increments. The interval between voltage steps was 3 seconds. Voltage dependence of inactivation was assessed by holding cells at various potentials from -160 to $-40 \mathrm{mV}$, followed by a $30-\mathrm{msec}$ test pulse to $-40 \mathrm{mV}$ to elicit $\mathrm{I}_{\mathrm{Na}}$. Recovery from inactivation was studied by holding cells at $-120 \mathrm{mV}$ and applying two 20 -msec test pulses $(\mathrm{S} 1, \mathrm{~S} 2)$ to $-40 \mathrm{mV}$ separated by increments of $1 \mathrm{msec}$ to a maximum S1-S2 interval of $50 \mathrm{msec}$. The S1-S1 interval was kept constant at 3 seconds.

Production and purification of fusion proteins. $\mathrm{cDNAs}$ for the AnkB MBD, spectrin-binding domain (SBD), and CTD were PCR amplified, subcloned into pGEX6P-1, and sequenced to confirm correct sequences. BL21(DE3)pLysS cells were transformed with the various AnkB pGEX6P1 constructs and grown overnight at $37^{\circ} \mathrm{C}$ in lysogeny broth (LB) supplemented with $0.1 \mathrm{~g} / \mathrm{L}$ ampicillin. The overnight cultures were subcultured for large-scale expression. The bacterial cells were grown to an OD of 0.6 and induced with $1 \mathrm{mM}$ isopropyl 1-thio- $\alpha$-D-galactopyranoside (IPTG) for 4 hours at $37^{\circ} \mathrm{C}$. Cells were centrifuged for 10 minutes at $8000 \times g$, resuspended in PBS, and frozen at $-20^{\circ} \mathrm{C}$ following resuspension. Lysis of cells was achieved by thawing. The crude extract was suspended in a solution of PBS, $1 \mathrm{mM}$ DTT, $1 \mathrm{mM}$ EDTA, $40 \mathrm{~g} / \mathrm{mL}$ 4-benzenesulfonyl fluoride hydrochloride (AEBSF), $10 \mathrm{~g} / \mathrm{mL}$ benzamide, and $10 \mathrm{~g} / \mathrm{mL}$ pepstatin. Lysates were homogenized by sonication and centrifuged to remove cellular debris, and the supernatant was incubated with glutathione-sepharose beads overnight at $4^{\circ} \mathrm{C}$. A small amount was separated by SDS-PAGE and stained with Coomassie blue to quantitate immobilized protein.

In vitro binding assays. In vitro direct binding assays were performed as previously described (17) using GST fusion proteins and ${ }^{35} \mathrm{~S}$-Met-labeled in vitro translation products. Reactions were performed at $4^{\circ} \mathrm{C}$ for 3 hours in a high-stringency binding buffer $(50 \mathrm{mM}$ Tris, pH 7.4, 1 mM EDTA, 1 mM EGTA, 500 mM NaCl, 0.1\% Triton $\mathrm{X}-100)$, washed 3 times with a high-stringency wash buffer $(1 \mathrm{M} \mathrm{NaCl}$ binding buffer), separated by SDS-PAGE, and visualized by phosphoimaging. In the non ${ }^{-35} \mathrm{~S}-\mathrm{Met}-\mathrm{labeled}$ GST pulldown, a $250-\mathrm{mM} \mathrm{NaCl}$ wash buffer was used.

Co-IP experiments. Mouse heart samples were flash-frozen in liquid nitrogen. Samples were resuspended in homogenization buffer and further homogenized by mechanical agitation with a Dounce homogenizer. Lysates were centrifuged for 15 minutes at 14,000 $\times g$. The resulting supernatant was incubated with $5 \mu \mathrm{l}$ anti-AnkB Ig at $4^{\circ} \mathrm{C}$ overnight. Each sample $(15 \mu \mathrm{g})$ was set aside to be used as an input loading control. After overnight incubation, the supernatant was removed from the beads, and the beads were washed 3 times with wash buffer ( $1 \times$ PBS, $0.1 \%$ Triton $\mathrm{X}-100$, and $150 \mathrm{~mm} \mathrm{NaCl}$ ) before immunoblotting with $\beta$-catenin (1:1000 IB, 1:100 IF; BD Biosciences, 610153).

SB-216763 prevention and reversal studies. Ank $2^{f / f l}$ and Ank2-cKO animals underwent daily intraperitoneal injections of $2.5 \mathrm{mg} / \mathrm{kg}$ SB-216763 dissolved in DMSO. Vehicle-treated animals were injected with an equivalent volume of DMSO alone. Low-volume HPLC syringes (Hamilton) were used, such that total injection volumes were less than $15 \mu \mathrm{L}$. Drug and vehicle injections were initiated in mice at 4 weeks of age in the prevention studies and performed until 12 weeks of age, whereas they commenced in mice at 12 weeks of age in the reversal studies and were continued for 4 weeks. In both instances, the echocardiography operator was blinded to genotype and treatment, whereas the administrator of therapy was not.

ANK2 analysis in the international multicenter ARVC cohort. An international multicenter cohort consisting of genotype-negative, Task Force Criteria-positive ARVC patients (meeting the criteria for "definite") underwent screening for the ANK2 gene. The cohort was made up of patients from the following institutions and registries: 4 North American and European medical centers: Johns Hopkins Medical Center (Baltimore, Maryland, USA); Academic Medical Centre (Amsterdam, Netherlands); Toronto General Hospital (Toronto, Ontario, Canada); University Hospital Linköping (Linköping, Sweden); and 2 registries (Familial Cardiomyopathy Registry, University of Colorado and the Canadian ARVC Registry). "Genotype negative" was defined as the absence of a suspected pathogenic mutation within 1 of the 5 desmosomal genes (PKP2, DSC2, DSG2, DSP, JUP) and TMEM43. Participant demographics and medical details were obtained through review of medical records.

All study participants underwent next-generation sequencing of the 53 exons comprising the ANK2 gene; sequencing and bioinformatic methodology for each site are provided in the Supplemental Methods. All identified rare ANK2 variants, defined as an allele frequency of less than $0.1 \%$ observed in gnomAD and anticipated to be functional (mis- 
sense, nonsense, and splice site variants, along with small insertion-deletions), were confirmed with Sanger sequencing. In silico prediction of the functional effects of missense mutations was examined using Polymorphism Phenotyping v2 (PolyPhen-2), Sorting Intolerant From Tolerant (SIFT), MutationTaster, and Combined Annotation-Dependent Depletion (CADD) (41-44). The collective prevalence of ANK2 rare variants identified in the multicenter ARVC cohort was compared with their cumulative prevalence and the cumulative prevalence of all ANK2 rare variants anticipated to be functional, as defined above, in gnomAD.

Statistics. Normally distributed continuous variables are presented as the mean \pm SD for clinical data and the mean \pm SEM for in vitro and in vivo analyses. $P$ values were determined with the unpaired, 2-tailed Student's $t$ test for single comparisons of normally distributed continuous variables, and the Fisher's exact test was used for comparisons of categorical values. Multiple comparisons were analyzed with ANOVA. Tukey's test was used for post hoc analyses. If the data distribution failed normality tests with the Shapiro-Wilk test, a rank-based ANOVA and Dunn's multiple comparisons test were performed. $P$ values in the survival study were determined using the log-rank test. Statistical analyses were performed using R and GraphPad Prism. $P$ values of less than 0.05 were considered statistically significant. At least 3 images were gathered for IF images from proband patient samples.

Study approval. The human aspects of the study were performed as part of a protocol approved by the research ethics boards of the UCSF Committee on Human Research and the collaborating institutions. All living human study participants provided written informed consent, and use of tissues from deceased individuals required consent from their next of kin. Human cardiac specimens were obtained from the cardiac tissue bank of the Department of Pathology at the University Medical Center (Utrecht, Netherlands), and the scientific advisory board of the University Medical Center Utrecht (Biobank) approved this component of the study. Animal procedures were approved and conducted in accordance with the IACUC of The Ohio State University. The use of mice conformed to guidelines set forth in the NIH's Guide for the Care and Use of Laboratory Animals (National Academies Press, 2011).

\section{Author contributions}

JDR, MMS, and PJM conceived, designed, and directed the study. NM, ERL, HM, MR, SK, SLS, MH, and CJMVO performed experiments and collected and analyzed data. CFK, TABVV, TJH, and PJM conceived experiments and directed results and strategy. JDR, RMH, CAJ, MHG, ADK, A.C. Sturm, MAA, ETH, SLG, RWD, MAR, TTK, SA, MF, MRGT, GS, KM, CT, BM, AC, BN, DTN, FIM,
NS, EML, MPVDB, DAS, JFR, AKG, A.C. Skanes, AST, MG, RAH, AAMW, JSH, PMLJ, LM, JPVT, HC, DPJ, and MMS acquired and analyzed clinical and/or genetic data. RMH, ERL, CAJ, CFK, MHG, ADK, A.C. Sturm, MAA, ETH, SLG, RWD, MAR, TTK, SA, MF, DAC, MRGT, SLS, MH, CJMVO, GS, KM, CT, BM, AC, BN, DTN, FIM, NS, EML, MPVDB, DAS, JFR, PCU, AKG, A.C. Skanes, AST, MG, RAH, TABVV, AAMW, JSH, PMLJ, LM, LEW, JPVT, HC, DPJ, TJH, and MMS critically reviewed and revised the manuscript. RMH, MAA, DAC, PCU, AKG, and MMS provided human tissue. JDR, NM, and PJM drafted and finalized the manuscript.

\section{Acknowledgments}

This work was supported by the Marianne Barrie Philanthropic Fund (to JDR) and the Canadian Institutes of Health Research (RN332805; to ADK). TABV was supported by the Netherlands CardioVascular Research Initiative: the Dutch Heart Foundation, the Dutch Federation of University Medical Centers, the Netherlands Organisation for Health Research and Development, and the Royal Netherlands Academy of Sciences (CVON-PREDICT 2012-10). JPVT acknowledges funding from the Netherlands Cardiovascular Research Initiative, an initiative supported by the Dutch Heart Foundation (CVON2012-10 PREDICT CVON2018-30 PREDICT2 and CVON2015-12 eDETECT). CAJ was supported by a visitor's travel grant from the Netherlands Organization for Scientific Research (NWO) (040.11.586). HC received funding from the Fondation Leducq (16 CVD 02). The Johns Hopkins ARVD/C Program is supported by the Dr. Francis P. Chiramonte Private Foundation; the Leyla Erkan Family Fund for ARVD Research; the Dr. Satish, Rupal; and Robin Shah ARVD Fund at Johns Hopkins, the Bogle Foundation, the Healing Hearts Foundation; the Campanella Family; the Patrick J. Harrison Family; the Peter French Memorial Foundation; and the Wilmerding Endowments. The authors are supported by NIH grants HL135754, HL134824, HL139348, HL135096, and HL114383 (to PJM); HL135096, HL134824, and HL114893 (to TJH); HL137331 (to ERL); HL137325 (to NPM); 2UM1HG006542 (to NS); and UL1 TR 001079 (to HC). This work is supported by a grant from the Ohio State Frick Center and JB Project (to PJM).

Address correspondence to: Peter J. Mohler, 110G DHLRI, 473 West 12th Avenue, Columbus, Ohio 43210, USA. Phone: 614.292.5019; Email: peter.mohler@osumc.edu. Or to: Jason D. Roberts, 339 Windermere Road, London, Ontario, Canada, N6A 5A5. Phone: 519.663.3746 ext.34526; Email: jason.roberts@lhsc.on.ca.
1. Corrado D, Link MS, Calkins H. Arrhythmogenic right ventricular cardiomyopathy. $N$ Engl J Med. 2017;376(15):1489-1490.

2. Sen-Chowdhry S, et al. Left-dominant arrhythmogenic cardiomyopathy: an underrecognized clinical entity. J Am Coll Cardiol. 2008;52(25):2175-2187.

3. Corrado D, et al. Treatment of arrhythmogenic right ventricular cardiomyopathy/dysplasia: an International Task Force consensus statement. Circulation. 2015;132(5):441-453.

4. McKoy G, et al. Identification of a deletion in plakoglobin in arrhythmogenic right ventricular cardiomyopathy with palmoplantar keratoderma and woolly hair (Naxos disease). Lancet.
2000;355(9221):2119-2124.

5. Gerull B, et al. Mutations in the desmosomal protein plakophilin-2 are common in arrhythmogenic right ventricular cardiomyopathy. Nat Genet. 2004;36(11):1162-1164.

6. Awad MM, et al. DSG2 mutations contribute to arrhythmogenic right ventricular dysplasia/cardiomyopathy. Am JHum Genet. 2006;79(1):136-142.

7. Garcia-Gras E, et al. Suppression of canonical Wnt/beta-catenin signaling by nuclear plakoglobin recapitulates phenotype of arrhythmogenic right ventricular cardiomyopathy. J Clin Invest. 2006;116(7):2012-2021.

8. Chen SN, Gurha P, Lombardi R, Ruggiero A, Willerson JT, Marian AJ. The hippo pathway is activated and is a causal mechanism for adipogenesis in arrhythmogenic cardiomyopathy. Circ Res. 2014;114(3):454-468.

9. Delmar M, McKenna WJ. The cardiac desmosome and arrhythmogenic cardiomyopathies: from gene to disease. Circ Res. 2010;107(6):700-714.

10. Marcus FI, et al. Right ventricular dysplasia: a report of 24 adult cases. Circulation. 1982;65(2):384-398.

11. Mohler PJ, et al. Ankyrin-B mutation causes type 4 long-QT cardiac arrhythmia and sudden cardiac death. Nature. 2003;421(6923):634-639.

12. Mohler PJ, et al. A cardiac arrhythmia syndrome caused by loss of ankyrin-B function. Proc Nat Acad Sci U S A. 2004;101(24):9137-9142. 
13. Cunha SR, et al. Defects in ankyrin-based membrane protein targeting pathways underlie atrial fibrillation. Circulation. 2011;124(11):1212-1222.

14. Le Scouarnec S, et al. Dysfunction in ankyrin-Bdependent ion channel and transporter targeting causes human sinus node disease. Proc Natl Acad SciU S A. 2008;105(40):15617-15622.

15. Scotland P, Zhou D, Benveniste H, Bennett V. Nervous system defects of AnkyrinB (-/-) mice suggest functional overlap between the cell adhesion molecule L1 and 440-kD AnkyrinB in premyelinated axons. J Cell Biol. 1998;143(5):1305-1315.

16. Dalal D, et al. Morphologic variants of familial arrhythmogenic right ventricular dysplasia/ cardiomyopathy a genetics-magnetic resonance imaging correlation study. J Am Coll Cardiol. 2009;53(15):1289-1299.

17. Lek M, et al. Analysis of protein-coding genetic variation in 60,706 humans. Nature. 2016;536(7616):285-291.

18. Kirchhof $\mathrm{P}$, et al. Age- and training-dependent development of arrhythmogenic right ventricular cardiomyopathy in heterozygous plakoglobin-deficient mice. Circulation. 2006;114(17):1799-1806.

19. Cerrone M, et al. Plakophilin-2 is required for transcription of genes that control calcium cycling and cardiac rhythm. Nat Commun. 2017;8(1):106.

20. Chelko SP, et al. Central role for GSK3 $\beta$ in the pathogenesis of arrhythmogenic cardiomyopathy. JCI Insight. 2016;1(5):e85923.

21. Moncayo-Arlandi J, et al. Molecular disturbance underlies to arrhythmogenic cardiomyopathy induced by transgene content, age and exercise in a truncated PKP2 mouse model. Hum Mol Genet. 2016;25(17):3676-3688.

22. Cruz FM, et al. Exercise triggers ARVC phenotype in mice expressing a disease-causing mutated version of human plakophilin-2. J Am Coll Cardiol. 2015;65(14):1438-1450.

23. Krusche CA, et al. Desmoglein 2 mutant mice develop cardiac fibrosis and dilation. Basic Res Cardiol. 2011;106(4):617-633.
24. Asimaki A, et al. A new diagnostic test for arrhythmogenic right ventricular cardiomyopathy. N Engl JMed. 2009;360(11):1075-1084.

25. Noorman M, et al. Remodeling of the cardiac sodium channel, connexin 43 , and plakoglobin at the intercalated disk in patients with arrhythmogenic cardiomyopathy. Heart Rhythm. 2013;10(3):412-419.

26. Asimaki A, et al. Identification of a new modulator of the intercalated disc in a zebrafish model of arrhythmogenic cardiomyopathy. Sci Transl Med. 2014;6(240):240ra74

27. Swayne LA, et al. Novel variant in the ANK2 membrane-binding domain is associated with ankyrin-B syndrome and structural heart disease in a First Nations population with a high rate of long QT syndrome. Circ Cardiovasc Genet. 2017;10(1):e001537.

28. Marcus FI, et al. Diagnosis of arrhythmogenic right ventricular cardiomyopathy/dysplasia: proposed modification of the task force criteria. Circulation. 2010;121(13):1533-1541.

29. Wolfe RS. My kind of biology. Annu Rev Microbiol. 1991;45:1-35.

30. Little SC, et al. Protein phosphatase 2 A regulatory subunit B56 $\alpha$ limits phosphatase activity in the heart. Sci Signal. 2015;8(386):ra72.

31. Zhou J, et al. Upregulation of gamma-catenin compensates for the loss of beta-catenin in adult cardiomyocytes. Am J Physiol Heart Circ Physiol. 2007;292(1):H270-H276.

32. James CA, et al. Exercise increases age-related penetrance and arrhythmic risk in arrhythmogenic right ventricular dysplasia/cardiomyopathy-associated desmosomal mutation carriers. J Am Coll Cardiol. 2013;62(14):1290-1297.

33. Xu T, et al. Compound and digenic heterozygosity contributes to arrhythmogenic right ventricular cardiomyopathy. J Am Coll Cardiol. 2010;55(6):587-597.

34. Sen-Chowdhry S, Syrris P, Pantazis A, Quarta G, McKenna WJ, Chambers JC. Mutational heterogeneity, modifier genes, and environmental influences contribute to phenotypic diversity of arrhythmogenic cardiomyopathy. Circ Cardiovasc Genet. 2010;3(4):323-330.

35. Haggerty CM, et al. Electronic health record phenotype in subjects with genetic variants associated with arrhythmogenic right ventricular cardiomyopathy: a study of 30,716 subjects with exome sequencing. Genet Med. 2017;19(11):1245-1252.

36. Hall CL, Sutanto H, Dalageorgou C, McKenna WJ, Syrris P, Futema M. Frequency of genetic variants associated with arrhythmogenic right ventricular cardiomyopathy in the genome aggregation database. Eur J Hum Genet. 2018;26(9):1312-1318.

37. Sawant AC, et al. Exercise has a disproportionate role in the pathogenesis of arrhythmogenic right ventricular dysplasia/cardiomyopathy in patients without desmosomal mutations. JAm Heart Assoc. 2014;3(6):e001471.

38. Musa $\mathrm{H}$, et al. Common human ANK2 variant confers in vivo arrhythmia phenotypes. Heart Rhythm. 2016;13(9):1932-1940.

39. Huq AJ, et al. A novel mechanism for human cardiac ankyrin-B syndrome due to reciprocal chromosomal translocation. Heart Lung Circ. 2017;26(6):612-618.

40. Cheng HW, et al. Assessment of right ventricular structure and function in mouse model of pulmonary artery constriction by transthoracic echocardiography. J Vis Exp. 2014;(84):e51041.

41. Adzhubei IA, et al. A method and server for predicting damaging missense mutations. Nat Methods. 2010;7(4):248-249.

42. Kumar P, Henikoff S, Ng PC. Predicting the effects of coding non-synonymous variants on protein function using the SIFT algorithm. Nat Protoc. 2009;4(7):1073-1081.

43. Schwarz JM, Rödelsperger C, Schuelke M, Seelow D. MutationTaster evaluates disease-causing potential of sequence alterations. Nat Methods. 2010;7(8):575-576.

44. Kircher M, Witten DM, Jain P, O'Roak BJ, Cooper GM, Shendure J. A general framework for estimating the relative pathogenicity of human genetic variants. Nat Genet. 2014;46(3):310-315. 